\title{
Rare top decays as probes of flavorful Higgs bosons
}

\author{
Wolfgang Altmannshofer, Brian Maddock, ${ }^{\dagger}$ and Douglas Tuckler ${ }^{\ddagger}$ \\ Santa Cruz Institute for Particle Physics, University of California, Santa Cruz, California 95064, USA
}

(Received 16 May 2019; published 8 July 2019)

\begin{abstract}
We study a version of two Higgs doublet models with nonstandard flavor violation in the up quark sector. We find branching ratios for the rare top decays $t \rightarrow h c$ and $t \rightarrow h u$ that are within reach of current and future colliders, while other flavor constraints from rare $B$ decays and neutral $D$ meson mixing, as well as constraints from Higgs signal strength measurements remain under control. The most prominent collider signature of the considered setup is $p p \rightarrow t H \rightarrow t t \bar{c}$ providing continued motivation to search for samesign tops at the LHC as well as a simple framework to interpret these searches. As a by-product of our study, we provide updated Standard Model predictions for the rare top decays $\operatorname{BR}(t \rightarrow h c)_{\mathrm{SM}}=$ $\left(4.19_{-0.80}^{+1.08} \pm 0.16\right) \times 10^{-15}$ and $\mathrm{BR}(t \rightarrow h u)_{\mathrm{SM}}=\left(3.66_{-0.70}^{+0.94} \pm 0.67\right) \times 10^{-17}$ with the main uncertainties coming from higher order QCD and Cabibbo-Kobayashi-Maskawa matrix elements.
\end{abstract}

DOI: 10.1103/PhysRevD.100.015003

\section{INTRODUCTION}

Flavor-changing neutral current (FCNC) decays of the top quark appear at one loop in the Standard Model (SM) and are strongly suppressed by the Glashow-IliopoulosMaiani (GIM) mechanism [1] and the small mixing of the third generation quarks with the first and second generations. In particular, the branching ratios of the rare decays $t \rightarrow h c$ and $t \rightarrow h u$ in the SM are predicted to be $\mathcal{O}\left(10^{-15}\right)$ and $\mathcal{O}\left(10^{-17}\right)[2,3]$, respectively, which renders these processes unobservable in the foreseeable future [4-9]. Observation of such processes at current or planned colliders would be a clear signal of physics beyond the SM.

The mass of the top quark is close to the electroweak scale and large compared to the masses of the rest of the quarks which suggests that it is intimately connected with the mechanism of electroweak symmetry breaking (EWSB). By now it has been well established that the main source of mass generation for the weak gauge bosons and the third generation fermions is the vacuum expectation value (VEV) of the $125 \mathrm{GeV}$ Higgs boson discovered at the Large Hadron Collider (LHC) [10-15]. However, much less is known about the origin of mass for the first and second generations of fermions. Although constraints have been placed on the $125 \mathrm{GeV}$ Higgs coupling to the

\footnotetext{
*waltmann@ucsc.edu

†bmaddock@ucsc.edu

dtuckler@ucsc.edu
}

Published by the American Physical Society under the terms of the Creative Commons Attribution 4.0 International license. Further distribution of this work must maintain attribution to the author(s) and the published article's title, journal citation, and DOI. Funded by SCOAP . muon [16,17], direct measurements of its couplings to the first and second generation fermions are experimentally difficult, and it is presently unknown if the $125 \mathrm{GeV}$ Higgs gives mass to the light fermions. In addition, the masses of the fermions as well as the Cabibbo-Kobayashi-Maskawa (CKM) quark mixing matrix exhibit a hierarchical structure that is not a priori explained by the SM-this is known as the SM flavor puzzle.

The SM flavor puzzle can be partially addressed by introducing an additional source of EWSB that is responsible for generating mass for the first and second generation fermions, as proposed in [18] (see also [19-22]). Arguably the simplest realization of this scenario is a two Higgs doublet model (2HDM), in which one Higgs doublet (approximately identified as the $125 \mathrm{GeV}$ Higgs boson) couples mainly to the third generation, while the second Higgs doublet couples mainly to the first and second generations. The observed pattern of quark masses and mixing can be obtained by asserting suitable textures for the quark mass matrices, leading to a "flavorful" two Higgs doublet model (F2HDM). The Yukawa structure of the F2HDM can be for example generated via the flavorlocking mechanism [23,24], and the implied collider phenomenology has been discussed in detail in [25]. Variations of the original F2HDM with alternative flavor structures were explored in [26] (for additional recent work on extended Higgs sectors with nonstandard flavor structures see [27-44]).

In this work, we will explore the effects of the F2HDM on rare top quark decays $t \rightarrow h u$ and $t \rightarrow h c$, where $h$ is the SM-like Higgs. In previous studies, we considered setups where the CKM matrix originates in the down quark sector; i.e., the CKM matrix is largely given by the matrix that diagonalizes the down quark Yukawa couplings. Such a 
setup is a natural choice given the hierarchies in the down quark masses and the CKM matrix elements are comparable, $V_{u s} \sim m_{d} / m_{s}, V_{c b} \sim m_{s} / m_{b}, V_{u b} \sim m_{d} / m_{b}$. Such setups can lead to enhanced flavor-violating couplings of the Higgs bosons to down-type quarks, resulting in potentially interesting effects in $B$ meson oscillations and rare $B$ decays. Rare top decays, however, tend to be strongly suppressed. In this work we will instead explore setups in which the CKM matrix is generated in the up quark sector, which can lead to enhanced tree-level flavorviolating couplings of the Higgs bosons to up-type quarks. These couplings can produce branching ratios for the rare top quark decays $t \rightarrow h u$ and $t \rightarrow h c$ that are orders of magnitudes greater than the SM predictions, and can be within reach of current and future colliders.

Because the mass hierarchies in the up quark sector are rather different from those in the down quark sector, the flavor-locking mechanism $[23,24]$ is not suitable for generating the CKM matrix in the up sector. Thus, we will consider a scenario where the required up Yukawa textures are dynamically generated by a Froggatt-Nielsen- (FN) type mechanism [45].

The paper is structured as follows. In Sec. II we discuss F2HDMs with a CKM matrix that originates in the up sector and identify a setup that dynamically generates the required flavor structure of the up Yukawa using the FN mechanism. In Sec. III we consider the stringent constraints on the model from the rare decay $b \rightarrow s \gamma$. In Sec. IV we first update the SM predictions for the branching ratios of the rare decays $t \rightarrow h u$ and $t \rightarrow h c$. We then study how these decays are affected by the tree-level flavor-changing Higgs couplings $h t c$ and $h t u$ and compare the F2HDM predictions for the $t \rightarrow h u$ and $t \rightarrow h c$ branching ratios with existing and expected experimental sensitivities. In Sec. V we discuss related effects of the model on neutral $D$ meson mixing and the collider phenomenology of the heavy Higgs bosons, identifying features that are different from the down-type F2HDMs studied in $[25,26]$. We conclude in Sec. VI.

\section{FLAVORFUL 2HDMS WITH UP-SECTOR CKM}

In a generic $2 \mathrm{HDM}$ the interactions of the two Higgs doublets, $\Phi$ and $\Phi^{\prime}$ (with VEVs $v$ and $v^{\prime}$ ), with the SM quarks and leptons are described by the Yukawa Lagrangian

$$
\begin{aligned}
-\mathcal{L}_{\mathrm{Yuk}}= & \sum_{i, j}\left(\lambda_{i j}^{u}\left(\bar{Q}_{i} u_{j}\right) \tilde{\Phi}+\lambda_{i j}^{d}\left(\bar{Q}_{i} d_{j}\right) \Phi+\lambda_{i j}^{e}\left(\bar{\ell}_{i} e_{j}\right) \Phi\right. \\
& \left.+\lambda_{i j}^{\prime \prime}\left(\bar{Q}_{i} u_{j}\right) \tilde{\Phi}^{\prime}+\lambda_{i j}^{\prime d}\left(\bar{Q}_{i} d_{j}\right) \Phi^{\prime}+\lambda_{i j}^{\prime e}\left(\bar{l}_{i} e_{j}\right) \Phi^{\prime}\right) \\
& + \text { H.c. }
\end{aligned}
$$

where $\tilde{\Phi}^{(\prime)}=i \sigma_{2}\left(\Phi^{(\prime)}\right)^{*}, Q_{i}, \ell_{i}$ are the three generations of the left-handed quark and lepton doublets, and $u_{i}, d_{i}, e_{i}$ are the three generations of right-handed up quark, down quark, and charged lepton singlets. Generically, the Yukawa matrices $\lambda^{(\prime) u, d, \ell}$ will contain off-diagonal entries that generate flavor-violating processes at tree level. In order to avoid tensions with low energy flavor constraints, one often imposes a discrete $\mathbb{Z}_{2}$ symmetry on the Higgs and quark fields such that the couplings of the Higgs bosons are flavor diagonal, leading to the well-studied 2HDMs with natural flavor conservation: type I, type II, flipped, and lepton specific [46].

A "flavorful" 2HDM, as introduced in [18,26], does not impose these discrete symmetries and instead assumes that one set of the Yukawa couplings is rank 1, preserving a $U(2)^{5}$ symmetry acting on the first two generations that is minimally broken by the second set of Yukawa couplings. In this way, flavor transitions between the first and second generations are protected and appear only at second order as an effective transition. In [26] four such models we identified, that, in analogy to the models with $\mathbb{Z}_{2}$ symmetry, were denoted by type IB, type IIB, flipped $\mathrm{B}$, and leptonspecific B 2HDMs. In Table I we summarize which Higgs boson is primarily responsible for generating the masses of each fermion.

In addition to reproducing the observed quark masses, the F2HDMs also need to accommodate the CKM quark mixing matrix. The CKM matrix arises from the mismatch of rotations of left-handed up- and down-type quarks when rotating into the quark mass eigenstate basis. The CKM matrix can originate dominantly from the rotations in the up quark sector or from those in the down quark sector. In previous studies [18,24-26] the CKM matrix was generated in the down quark sector. In this work we will instead generate the CKM matrix in the up quark sector.

Since the hierarchies in the up quark masses are different from those in the CKM matrix, the flavor-locking mechanism is not suitable for generating appropriate Yukawa textures for an "up-type" F2HDM. We will therefore use the Froggatt-Nielsen mechanism, which explains the hierarchy of quark masses and mixing by introducing an Abelian flavor symmetry-which we will denote by $U(1)_{\mathrm{FN}}$ - that distinguishes different fermion flavors. The flavor symmetry is broken by the VEV of a SM-singlet scalar field, $S$, that carries a $U(1)_{\mathrm{FN}}$ charge $Q_{S}=+1$. This breaking is communicated to the SM fermions by higher-dimensional operators leading to Yukawa couplings that are suppressed by powers of a small symmetry-breaking parameter

TABLE I. Dominant source of mass for the SM fermions in F2HDMs.

\begin{tabular}{lcccccc}
\hline \hline Model & $u, c$ & $t$ & $d, s$ & $b$ & $e, \mu$ & $\tau$ \\
\hline Type 1B & $\Phi^{\prime}$ & $\Phi$ & $\Phi^{\prime}$ & $\Phi$ & $\Phi^{\prime}$ & $\Phi$ \\
Type 2B & $\Phi^{\prime}$ & $\Phi$ & $\Phi$ & $\Phi^{\prime}$ & $\Phi$ & $\Phi^{\prime}$ \\
Flipped B & $\Phi^{\prime}$ & $\Phi$ & $\Phi$ & $\Phi^{\prime}$ & $\Phi^{\prime}$ & $\Phi$ \\
Lepton-specific B & $\Phi^{\prime}$ & $\Phi$ & $\Phi^{\prime}$ & $\Phi$ & $\Phi$ & $\Phi^{\prime}$ \\
\hline \hline
\end{tabular}


$\epsilon=\langle S\rangle / \Lambda_{S}$, where $\Lambda_{S} \gg v, v^{\prime}$ is the scale associated with the breaking of $U(1)_{\mathrm{FN}}$. In the resulting effective theory, the Yukawa Lagrangian is given by ${ }^{1}$

$$
-\mathcal{L}_{\text {Yuk }}^{\text {eff }} \supset \sum_{i, j}\left[\left(\frac{\langle S\rangle}{\Lambda_{S}}\right)^{\left|x_{i j}^{u}\right|}\left(\bar{Q}_{i} u_{j}\right) \tilde{\Phi}+\left(\frac{\langle S\rangle}{\Lambda_{S}}\right)^{\left|x_{i j}^{\prime u}\right|}\left(\bar{Q}_{i} u_{j}\right) \tilde{\Phi}^{\prime}\right],
$$

where the powers $x_{i j}^{(\prime) u}$ are determined from the charge assignments of the Higgs and quark fields under $U(1)_{\mathrm{FN}}$, and we have omitted model-dependent prefactors of $O(1)$. In terms of the parameter $\epsilon \approx 0.22$ we aim for the following relations for the quark masses and the CKM matrix elements

$$
\begin{aligned}
& \frac{m_{u}}{v_{\mathrm{w}}} \sim \epsilon^{8}, \quad \frac{m_{c}}{v_{\mathrm{w}}} \sim \epsilon^{3}, \quad \frac{m_{t}}{v_{\mathrm{w}}} \sim \epsilon^{0}, \\
& \frac{m_{d}}{v_{\mathrm{w}}} \sim \epsilon^{7}, \quad \frac{m_{s}}{v_{\mathrm{w}}} \sim \epsilon^{5}, \quad \frac{m_{b}}{v_{\mathrm{w}}} \sim \epsilon^{3}, \\
& \left|V_{u s}\right| \sim \lambda_{c} \sim \epsilon, \quad\left|V_{u b}\right| \sim \lambda_{c}^{3} \sim \epsilon^{3}, \quad\left|V_{c b}\right| \sim \lambda_{c}^{2} \sim \epsilon^{2},
\end{aligned}
$$

with the electroweak breaking VEV in the SM $v_{\mathrm{w}}=$ $\sqrt{v^{2}+v^{\prime 2}} \simeq 246 \mathrm{GeV}$ and the Cabibbo angle $\lambda_{c} \simeq 0.22$.

In order to obtain the rank 1 structure of the Yukawa couplings of $\Phi$ required by the F2HDM scenario, we introduce an additional $U(1)^{\prime}$ symmetry. A rank 1 Yukawa coupling $\lambda_{u}$ and simultaneous generation of the CKM matrix by $\lambda_{u}^{\prime}$ is possible by charging either the left-handed quark doublet $Q_{3}$ or the right-handed top $U_{3}$ under the additional symmetry. 2HDMs with a right-handed top that is singled out by a symmetry have been discussed e.g., in $[29,37]$. Here we follow the second option and set the $U(1)^{\prime}$ charges $Q_{\Phi}^{\prime}=-Q_{Q_{3}}^{\prime}=+1$, while leaving the right-handed top uncharged. We will see that this leads to highly predictive scenarios.

The remaining charge assignments depend on the type of F2HDM under consideration as well as on the value of the parameter $\tan \beta=v / v^{\prime}$ that can provide part of the fermion mass hierarchies. We restrict the following discussion to the quark sector. The extension to charged leptons is straightforward.

\section{A. Type IB and lepton-specific B}

In these types, the coupling of $\Phi$ to both up-type and down-type quarks is rank 1 . Given our choice of $U(1)^{\prime}$ charges discussed above, the charge of the right-handed bottom quark is required to be $Q_{d_{3}}^{\prime}=-2$, while all other quarks remain uncharged under the $U(1)^{\prime}$. For a given value of $\tan \beta$, the scaling in (3) fixes all $U(1)_{\mathrm{FN}}$ FroggattNielsen charges up a few discrete choices. In Table II we show all inequivalent charge assignments in the cases $\tan \beta \sim 1 / \epsilon \sim 5$ and $\tan \beta \sim 1 / \epsilon^{2} \sim 25$. The charge assignments lead to the following structure for the Yukawa couplings

$v \lambda_{u} \sim v_{\mathrm{w}}\left(\begin{array}{ccc}0 & 0 & 0 \\ 0 & 0 & 0 \\ \epsilon^{|a|} & \epsilon^{1} & 1\end{array}\right), \quad v^{\prime} \lambda_{u}^{\prime} \sim v_{\mathrm{w}}\left(\begin{array}{ccc}\epsilon^{8} & \epsilon^{4} & \epsilon^{3} \\ \epsilon^{|b|} & \epsilon^{3} & \epsilon^{2} \\ 0 & 0 & 0\end{array}\right)$,

$v \lambda_{d} \sim v_{\mathrm{w}}\left(\begin{array}{ccc}0 & 0 & 0 \\ 0 & 0 & 0 \\ 0 & 0 & \epsilon^{3}\end{array}\right), \quad v^{\prime} \lambda_{d}^{\prime} \sim v_{\mathrm{w}}\left(\begin{array}{ccc}\epsilon^{7} & \epsilon^{6} & 0 \\ \epsilon^{|c|} & \epsilon^{5} & 0 \\ 0 & 0 & 0\end{array}\right)$,

with the powers $|a|=5$ or 7 or $9,|b|=7$ or 9 , and $|c|=6$ or 8 , depending on the charge assignments and $\tan \beta$. It is easy to check that the diagonalization of the quark masses that are induced by these Yukawa couplings leads to a CKM matrix with the right texture that is indeed dominantly generated from the up quark rotation. Interestingly, the powers $|a|,|b|$, and $|c|$ are not observable in the IR. More importantly, in the quark mass eigenstate basis, the flavor structure of all couplings of the Higgs bosons is entirely determined by the known quark masses and CKM elements. The couplings of the physical Higgs mass eigenstates $h, H, A, H^{ \pm}$to the quarks can be parametrized by

$$
\begin{aligned}
-\mathcal{L}_{\text {Yuk }} \supset & \sum_{i, j}\left(\bar{d}_{i} P_{R} d_{j}\right)\left(h\left(Y_{h}^{d}\right)_{i j}+H\left(Y_{H}^{d}\right)_{i j}-i A\left(Y_{A}^{d}\right)_{i j}\right)+\text { H.c. } \\
& +\sum_{i, j}\left(\bar{u}_{i} P_{R} u_{j}\right)\left(h\left(Y_{h}^{u}\right)_{i j}+H\left(Y_{H}^{u}\right)_{i j}+i A\left(Y_{A}^{u}\right)_{i j}\right)+\text { H.c. } \\
& +\sqrt{2} \sum_{i, j}\left(\left(\bar{d}_{i} P_{R} u_{j}\right) H^{-}\left(Y_{ \pm}^{u}\right)_{i j}-\left(\bar{u}_{i} P_{R} d_{j}\right) H^{+}\left(Y_{ \pm}^{d}\right)_{i j}\right)+\text { H.c. }
\end{aligned}
$$

For all charge assignments we find for the up quark couplings

\footnotetext{
${ }^{1}$ Here we only describe the up quark sector, but an analogous discussion applies for down quarks and leptons as well.
} 
TABLE II. Charges of the Froggatt-Nielsen scalar $S$, the two Higgs doublets $\Phi$ and $\Phi^{\prime}$, and quark fields under the $U(1)_{\mathrm{FN}}$ and $U(1)^{\prime}$ symmetries in the type IB and lepton-specific B models for the two choices of $\tan \beta \sim 1 / \epsilon$ and $\tan \beta \sim 1 / \epsilon^{2}$.

\begin{tabular}{llcccccccccccc}
\hline \hline & $S$ & $\Phi$ & $\Phi^{\prime}$ & $\bar{Q}_{1}$ & $\bar{Q}_{2}$ & $\bar{Q}_{3}$ & $u_{1}$ & $u_{2}$ & $u_{3}$ & $d_{1}$ & $d_{2}$ & $d_{3}$ \\
\hline $\tan \beta \sim 1 / \epsilon$ & $U(1)_{\mathrm{FN}}$ & 1 & 0 & 0 & 2 & 1 & 0 & 5 or -9 & 1 & 0 & 4 or -8 & 3 & \pm 3 \\
$\tan \beta \sim 1 / \epsilon^{2}$ & $U(1)_{\mathrm{FN}}$ & 1 & 0 & 0 & 1 & 0 & 0 & 5 or -7 & 1 & 0 & 4 or -6 & 3 & \pm 3 \\
& $U(1)^{\prime}$ & 0 & 1 & 0 & 0 & 0 & 1 & 0 & 0 & 0 & 0 & 0 & -2 \\
\hline \hline
\end{tabular}

$v_{\mathrm{w}} Y_{h}^{u}=\frac{c_{\alpha}}{s_{\beta}}\left(\begin{array}{ccc}0 & 0 & 0 \\ 0 & 0 & 0 \\ 0 & 0 & m_{t}\end{array}\right)-\frac{s_{\alpha}}{c_{\beta}}\left(\begin{array}{ccc}m_{u} & 0 & 0 \\ 0 & m_{c} & 0 \\ 0 & 0 & 0\end{array}\right)+\frac{c_{\beta-\alpha}}{s_{\beta} c_{\beta}} \hat{M}_{u}$,

$$
v_{\mathrm{w}} Y_{H}^{u}=\frac{1}{t_{\beta}} \frac{s_{\alpha}}{c_{\beta}}\left(\begin{array}{ccc}
0 & 0 & 0 \\
0 & 0 & 0 \\
0 & 0 & m_{t}
\end{array}\right)+t_{\beta} \frac{c_{\alpha}}{s_{\beta}}\left(\begin{array}{ccc}
m_{u} & 0 & 0 \\
0 & m_{c} & 0 \\
0 & 0 & 0
\end{array}\right)-\frac{s_{\beta-\alpha}}{s_{\beta} c_{\beta}} \hat{M}_{u},
$$

$$
v_{\mathrm{w}} Y_{A}^{u}=-\frac{1}{t_{\beta}}\left(\begin{array}{ccc}
0 & 0 & 0 \\
0 & 0 & 0 \\
0 & 0 & m_{t}
\end{array}\right)+t_{\beta}\left(\begin{array}{ccc}
m_{u} & 0 & 0 \\
0 & m_{c} & 0 \\
0 & 0 & 0
\end{array}\right)-\frac{1}{s_{\beta} c_{\beta}} \hat{M}_{u},
$$

$$
\begin{aligned}
v_{\mathrm{w}} Y_{ \pm}^{u}= & -\frac{1}{t_{\beta}}\left(\begin{array}{ccc}
0 & 0 & 0 \\
0 & 0 & 0 \\
m_{u} V_{u b}^{*} & m_{c} V_{c b}^{*} & m_{t} V_{t b}^{*}
\end{array}\right) \\
& +t_{\beta}\left(\begin{array}{ccc}
m_{u} V_{u d}^{*} & m_{c} V_{c d}^{*} & m_{t} V_{t d}^{*} \\
m_{u} V_{u s}^{*} & m_{c} V_{c s}^{*} & m_{t} V_{t s}^{*} \\
0 & 0 & 0
\end{array}\right),
\end{aligned}
$$

and for the down quark couplings

$$
\begin{aligned}
& v_{\mathrm{w}} Y_{h}^{d}=\frac{c_{\alpha}}{s_{\beta}}\left(\begin{array}{ccc}
0 & 0 & 0 \\
0 & 0 & 0 \\
0 & 0 & m_{b}
\end{array}\right)-\frac{s_{\alpha}}{c_{\beta}}\left(\begin{array}{ccc}
m_{d} & 0 & 0 \\
0 & m_{s} & 0 \\
0 & 0 & 0
\end{array}\right), \\
& v_{\mathrm{w}} Y_{H}^{d}=\frac{1}{t_{\beta}} \frac{s_{\alpha}}{c_{\beta}}\left(\begin{array}{ccc}
0 & 0 & 0 \\
0 & 0 & 0 \\
0 & 0 & m_{b}
\end{array}\right)+t_{\beta} \frac{c_{\alpha}}{s_{\beta}}\left(\begin{array}{ccc}
m_{d} & 0 & 0 \\
0 & m_{s} & 0 \\
0 & 0 & 0
\end{array}\right), \\
& v_{\mathrm{w}} Y_{A}^{d}=-\frac{1}{t_{\beta}}\left(\begin{array}{ccc}
0 & 0 & 0 \\
0 & 0 & 0 \\
0 & 0 & m_{b}
\end{array}\right)+t_{\beta}\left(\begin{array}{ccc}
m_{d} & 0 & 0 \\
0 & m_{s} & 0 \\
0 & 0 & 0
\end{array}\right),
\end{aligned}
$$

$$
v_{\mathrm{w}} Y_{ \pm}^{d}=-\frac{1}{t_{\beta}}\left(\begin{array}{ccc}
0 & 0 & V_{u b} m_{b} \\
0 & 0 & V_{c b} m_{b} \\
0 & 0 & V_{t b} m_{b}
\end{array}\right)+t_{\beta}\left(\begin{array}{ccc}
V_{u d} m_{d} & V_{u s} m_{s} & 0 \\
V_{c d} m_{d} & V_{c s} m_{s} & 0 \\
V_{t d} m_{d} & V_{t s} m_{s} & 0
\end{array}\right) .
$$

The angle $\alpha$ in the above expressions parametrizes the mixing between the neutral scalar Higgs bosons $h$ and $H$. The mass matrix $\hat{M}_{u}$ that enters the up quark couplings is given by

$$
\hat{M}_{u}=\left(\begin{array}{ccc}
m_{u}\left|V_{u b}\right|^{2} & m_{c} V_{u b} V_{c b}^{*} & m_{t} V_{u b} V_{t b}^{*} \\
m_{u} V_{c b} V_{u b}^{*} & m_{c}\left|V_{c b}\right|^{2} & m_{t} V_{c b} V_{t b}^{*} \\
m_{u} V_{t b} V_{u b}^{*} & m_{c} V_{t b} V_{c b}^{*} & -m_{t}\left(\left|V_{c b}\right|^{2}+\left|V_{u b}\right|^{2}\right)
\end{array}\right) .
$$

The proof that the flavor structure of the Higgs couplings in the type IB and lepton-specific B models is indeed entirely determined by known quark masses and CKM elements is given in the Appendix A. Note that the neutral Higgs couplings are flavor diagonal in the down sector. Therefore, there are no tree-level contributions to e.g., $B$ and $K$ meson oscillations and rare $B$ meson decays. In the up sector, the neutral Higgs couplings are flavor violating but the amount of flavor violation is controlled by the CKM matrix. Remarkably, the only free parameters in the couplings are $\tan \beta$ and the Higgs mixing angle $\alpha$, making the type IB and lepton-specific B models with up-sector CKM highly predictive.

\section{B. Type IIB and flipped B}

In these types, the coupling of $\Phi$ to the up-type quarks and the coupling of $\Phi^{\prime}$ to the down-type quarks are rank 1 . We find that with our $U(1)^{\prime} \times U(1)_{\mathrm{FN}}$ setup, it is not possible to construct Yukawa matrices for the down-type quarks that exactly mirror the couplings in Eq. (4b), but with the role of $\lambda^{d}$ and $\lambda^{\prime d}$ exchanged.

However, we find that the $\lambda^{\prime d}$ couplings can still be made rank 1, with a consistent flavor structure as long as $\tan \beta \sim 1 / \epsilon \sim 5$. In contrast to the type IB and leptonspecific B setups discussed above, we find that $\lambda^{d}$ and $\lambda^{\prime d}$ necessarily contain mixing between the third and the first two generations. One example set of charges is given in Table III which leads to 
TABLE III. Example charges of the Froggatt-Nielsen scalar $S$, the two Higgs doublets $\Phi$ and $\Phi^{\prime}$, and quark fields under the $U(1)_{\mathrm{FN}}$ and $U(1)^{\prime}$ symmetries in the type IIB and lepton-specific B models for $\tan \beta \sim 1 / \epsilon$.

\begin{tabular}{|c|c|c|c|c|c|c|c|c|c|c|c|c|c|}
\hline & & $S$ & $\Phi$ & $\Phi^{\prime}$ & $\bar{Q}_{1}$ & $\bar{Q}_{2}$ & $\bar{Q}_{3}$ & $u_{1}$ & $u_{2}$ & $u_{3}$ & $d_{1}$ & $d_{2}$ & $d_{3}$ \\
\hline $\tan \beta \sim 1 / \epsilon$ & $\begin{array}{l}U(1)_{\mathrm{FN}} \\
U(1)^{\prime}\end{array}$ & $\begin{array}{l}0 \\
0\end{array}$ & $\begin{array}{r}0 \\
+1\end{array}$ & $\begin{array}{r}0 \\
-1\end{array}$ & $\begin{array}{r}0 \\
+1\end{array}$ & $\begin{array}{l}+1 \\
+1\end{array}$ & $\begin{array}{l}+2 \\
-1\end{array}$ & $\begin{array}{r}-7 \\
0\end{array}$ & $\begin{array}{r}-3 \\
0\end{array}$ & $\begin{array}{r}-2 \\
0\end{array}$ & $\begin{array}{r}-7 \\
0\end{array}$ & $\begin{array}{r}-6 \\
0\end{array}$ & $\begin{array}{r}-4 \\
0\end{array}$ \\
\hline
\end{tabular}

$v \lambda_{u} \sim v_{\mathrm{w}}\left(\begin{array}{ccc}0 & 0 & 0 \\ 0 & 0 & 0 \\ \epsilon^{5} & \epsilon^{1} & \epsilon^{0}\end{array}\right), \quad v^{\prime} \lambda_{u}^{\prime} \sim v_{\mathrm{w}}\left(\begin{array}{ccc}\epsilon^{8} & \epsilon^{4} & \epsilon^{3} \\ \epsilon^{7} & \epsilon^{3} & \epsilon^{2} \\ 0 & 0 & 0\end{array}\right)$

$$
v \lambda_{d} \sim v_{\mathrm{w}}\left(\begin{array}{ccc}
\epsilon^{7} & \epsilon^{6} & \epsilon^{4} \\
\epsilon^{6} & \epsilon^{5} & \epsilon^{3} \\
0 & 0 & 0
\end{array}\right), \quad v^{\prime} \lambda_{d}^{\prime} \sim v_{\mathrm{w}}\left(\begin{array}{ccc}
0 & 0 & 0 \\
0 & 0 & 0 \\
\epsilon^{6} & \epsilon^{5} & \epsilon^{3}
\end{array}\right)
$$

The more generic structure of the down quark Yukawas implies that the CKM matrix is partly generated also from the rotations in the down sector. Correspondingly, in the type IIB and flipped B models only the generic scaling of the couplings of the physical Higgs bosons can be predicted. The precise values of the physical Higgs couplings depend on unknown $O(1)$ parameters.

As we will see in Sec. III, constraints from the rare decay $B \rightarrow X_{s} \gamma$ push the masses of the additional Higgs bosons to uninterestingly large values in the type IIB and flipped B models. We therefore forgo an in-depth discussion of constructing the mass matrices and couplings in those types.

\section{CONSTRAINTS FROM RARE B DECAYS}

As discussed in the previous section, in the type IB and lepton-specific B models the neutral Higgs bosons couple to down-type quarks in a flavor diagonal way. Many constraints from FCNCs in the down quark sector are therefore automatically avoided. There is one important exception: the $b \rightarrow s \gamma$ decay. We find that one-loop contributions from charged Higgs bosons can lead to sizable new physics (NP) effects in the $b \rightarrow s \gamma$ transition. $^{2}$ Both the SM prediction [47] and the experimental measurements of the $B \rightarrow X_{s} \gamma$ rate [48] have uncertainties of less than $10 \%$ and are in good agreement with each other, resulting in strong constraints on nonstandard effects.

\footnotetext{
${ }^{2}$ We also checked one-loop charged Higgs contributions to the $B_{s} \rightarrow \mu^{+} \mu^{-}$decay and tree-level charged Higgs contributions to the $B \rightarrow \tau \nu$ and $B \rightarrow D^{(*)} \tau \nu$ decays and found that they are negligible in regions of parameter space that are allowed by $b \rightarrow s \gamma$.
}

The new physics effects induced by charged Higgs loops can be described by modifications of the Wilson coefficients $C_{7}$ and $C_{8}$ of an effective Hamiltonian

$$
\mathcal{H}_{\mathrm{eff}}^{\mathrm{NP}}=-\frac{4 G_{F}}{\sqrt{2}} V_{t b} V_{t s}^{*} \frac{e^{2}}{16 \pi^{2}}\left(\Delta C_{7} Q_{7}+\Delta C_{8} Q_{8}\right)
$$

with the dipole operators

$$
Q_{7}=\frac{1}{e} m_{b}\left(\bar{s} \sigma_{\mu \nu} P_{R} b\right) F^{\mu \nu}, \quad Q_{8}=\frac{g_{s}}{e^{2}}\left(\bar{s} \sigma_{\mu \nu} T^{a} P_{R} b\right) G_{a}^{\mu \nu} .
$$

Using the results from [49] we find for the charged Higgs contribution in the type IB and lepton-specific B scenarios

$$
\begin{aligned}
\Delta C_{7} & =\frac{m_{t}^{2}}{m_{H^{ \pm}}^{2}} f_{7}\left(\frac{m_{t}^{2}}{m_{H^{ \pm}}^{2}}\right), \\
\Delta C_{8} & =\frac{m_{t}^{2}}{m_{H^{ \pm}}^{2}} f_{8}\left(\frac{m_{t}^{2}}{m_{H^{ \pm}}^{2}}\right) .
\end{aligned}
$$

In the type IIB and flipped B scenarios the Wilson coefficients are only determined up to model-dependent $O(1)$ factors

$$
\begin{gathered}
\Delta C_{7}=O(1) \times\left(\frac{m_{t}^{2}}{m_{H^{ \pm}}^{2}} g_{7}\left(\frac{m_{t}^{2}}{m_{H^{ \pm}}^{2}}\right)+\tan ^{2} \beta \frac{m_{t}^{2}}{m_{H^{ \pm}}^{2}} h_{7}\left(\frac{m_{t}^{2}}{m_{H^{ \pm}}^{2}}\right)\right), \\
\Delta C_{8}=O(1) \times\left(\frac{m_{t}^{2}}{m_{H^{ \pm}}^{2}} g_{8}\left(\frac{m_{t}^{2}}{m_{H^{ \pm}}^{2}}\right)+\tan ^{2} \beta \frac{m_{t}^{2}}{m_{H^{ \pm}}^{2}} h_{8}\left(\frac{m_{t}^{2}}{m_{H^{ \pm}}^{2}}\right)\right) .
\end{gathered}
$$

The loop functions $f_{7,8}, g_{7,8}$, and $h_{7,8}$ are given in Appendix B. Note that in our type IB and lepton-specific B scenarios the contributions are independent of $\tan \beta$, while the contributions in the type IIB and flipped B scenarios contain terms that are proportional to $\tan ^{2} \beta$ and can become extremely large in regions of parameter space with large $\tan \beta$. This is in contrast to $2 \mathrm{HDMs}$ with natural flavor conservation, where the contributions are proportional to $1 / \tan ^{2} \beta$ (type I and lepton specific) and independent of $\tan \beta$ (type II and flipped). 
Using the constraints on the Wilson coefficients from $b \rightarrow s \gamma$ transitions derived in [50] and taking into account one-loop renormalization group running between the electroweak scale and the $b$ scale, we find at the 95\% C.L.

$$
-0.032<\eta^{\frac{16}{23}} \Delta C_{7}+\frac{8}{3}\left(\eta^{\frac{14}{23}}-\eta^{\frac{16}{23}}\right) \Delta C_{8}<0.027,
$$

with $\eta=\alpha_{s}\left(m_{t}\right) / \alpha_{s}\left(m_{b}\right) \simeq 0.52$.

The corresponding constraints are shown in the plots of Fig. 1 in the charged Higgs mass $m_{H^{ \pm}}$vs $\tan \beta$ plane. In the case of the type IB and lepton-specific B models, we find a $\tan \beta$ independent bound on the charged Higgs mass of $m_{H^{ \pm}} \gtrsim 800 \mathrm{GeV}$. In the type IIB and flipped B models, we show as illustration the case where the free $O(1)$ parameters are set to exactly 1 . In these types of models, the $b \rightarrow s \gamma$ constraint is highly dependent on $\tan \beta$, e.g., $m_{H^{ \pm}} \gtrsim 800 \mathrm{GeV}$ for $\tan \beta=1$, but $m_{H^{ \pm}} \gtrsim 15 \mathrm{TeV}$ for $\tan \beta=10$. Varying the $O(1)$ coefficients shifts the exclusion line up or down by an order 1 factor.

Note that because of the $S U(2)_{L}$ gauge symmetry, the masses of the heavy scalar and pseudoscalar Higgs differ from the charged Higgs mass only by a small amount: $m_{H} \simeq m_{A} \simeq m_{H^{ \pm}}$with splittings of the order of $v^{2} / m_{H^{ \pm}}^{2} \lesssim 10 \%$. The bounds on the charged Higgs mass from $b \rightarrow s \gamma$ therefore hold approximately for the masses of the heavy scalar and pseudoscalar Higgs as well.

As discussed in Sec. II, for the purpose of generating the fermion mass hierarchy we have in mind values of $\tan \beta \sim$ $1 / \lambda_{c} \sim 5$ or $\tan \beta \sim 1 / \lambda_{c}^{2} \sim 25$. In the type IIB and flipped B models, we see that the $b \rightarrow s \gamma$ constraints therefore strongly disfavor Higgs bosons with masses at the $\mathrm{TeV}$ scale. This remains true even if we take into account

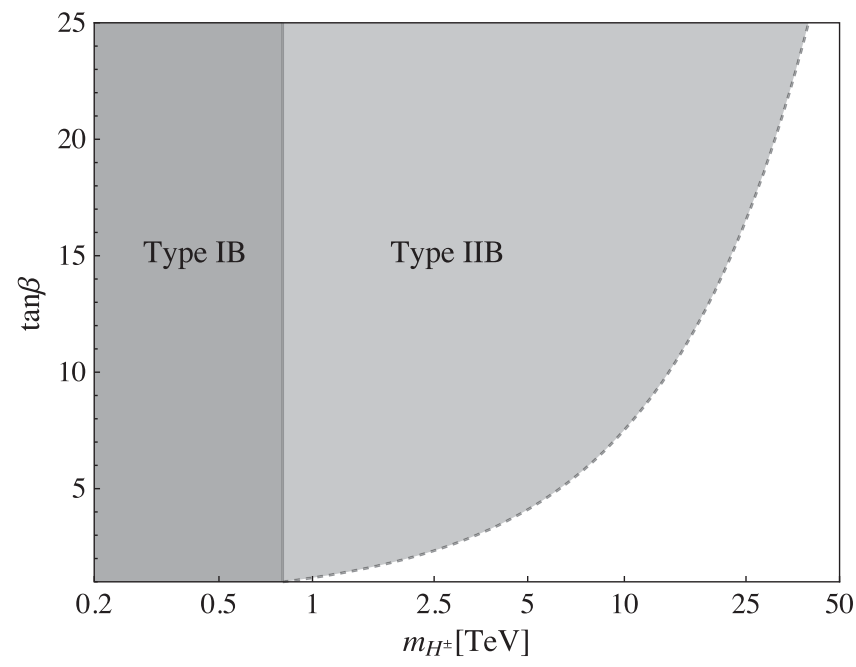

FIG. 1. Constraints from the $b \rightarrow s \gamma$ transition in the charged Higgs mass $m_{H^{ \pm}}$vs $\tan \beta$ plane. The dark gray region is excluded in the type IB and lepton-specific B scenarios at the 95\% C.L. The light gray region is excluded in the type IIB and flipped B scenarios at the $95 \%$ C.L. generous choices of the free $O(1)$ parameters. With this in mind we focus our remaining analysis on the type IB and lepton-specific B models.

\section{RARE TOP DECAYS}

In the SM, flavor-changing top quark decays $t \rightarrow h q$ are both loop and GIM suppressed, and are predicted to have very small branching ratios. Using the results from [51,52] for the partial widths $\Gamma(t \rightarrow h q)$ and normalizing to the $t \rightarrow W b$ decay width which dominates the total top width, we derive the following compact expression for the branching ratios

$$
\begin{aligned}
\operatorname{BR}(t & \rightarrow h q)_{\mathrm{SM}} \\
= & \frac{G_{F}^{2} m_{b}^{4}}{4 \pi^{4}}\left|V_{q b}\right|^{2} \frac{\left(1-m_{h}^{2} / m_{t, \mathrm{pole}}^{2}\right)^{2}}{\left(1-m_{W}^{2} / m_{t, \mathrm{pole}}^{2}\right)^{2}\left(1+2 m_{W}^{2} / m_{t, \mathrm{pole}}^{2}\right)} \\
& \times \mathcal{F}\left(\frac{m_{t}^{2}}{m_{W}^{2}}, \frac{m_{h}^{2}}{m_{W}^{2}}\right) .
\end{aligned}
$$

The branching ratio is suppressed by 4 powers of the bottom mass, as expected from GIM. We use the bottom $\overline{\mathrm{MS}}$ mass at the scale of the top $m_{b}\left(m_{t}\right)=2.73 \mathrm{GeV}$. Note that we are using the top pole mass in the phase space factors, but the top $\overline{\mathrm{MS}}$ mass in the loop function $\mathcal{F}$. The explicit expression for $\mathcal{F}$ is given in Appendix C. For central values of the Higgs mass $m_{h}=125.18 \mathrm{GeV}$ [53] and the top $\overline{\mathrm{MS}}$ mass $m_{t}\left(m_{t}\right)=163.4 \mathrm{GeV}$ (corresponding to a top pole mass of $m_{t, \text { pole }}=173.0 \mathrm{GeV}$ [53]) we find $F \simeq 0.48$. By far the largest uncertainties in the rare top branching ratios are due to the CKM factors $\left|V_{c b}\right|=(42.2 \pm 0.8) \times 10^{-3}$ [53] and $\left|V_{u b}\right|=(3.94 \pm 0.36) \times 10^{-3} \quad$ [53] and due to higher order QCD effects that we estimate by varying the renormalization scale of the bottom $\overline{\mathrm{MS}}$ mass $m_{b}(\mu)$ in the range $m_{t} / 2<\mu<2 m_{t}$. We obtain

$$
\begin{aligned}
& \mathrm{BR}(t \rightarrow h u)_{\mathrm{SM}}=\left(3.66_{-0.70}^{+0.94} \pm 0.67\right) \times 10^{-17}, \\
& \mathrm{BR}(t \rightarrow h c)_{\mathrm{SM}}=\left(4.19_{-0.80}^{+1.08} \pm 0.16\right) \times 10^{-15},
\end{aligned}
$$

where the first uncertainty is due to the variation of the renormalization scale and the second is due to the CKM matrix elements. The current strongest experimental bounds on these decays are obtained by the ATLAS experiment, using an integrated luminosity of $36 \mathrm{fb}^{-1}$ of $p p$ collision data with $\sqrt{s}=13 \mathrm{TeV}$ in multilepton final state searches [4], and read

$$
\begin{aligned}
& \mathrm{BR}(t \rightarrow h u)<0.12 \%, \\
& \mathrm{BR}(t \rightarrow h c)<0.11 \% .
\end{aligned}
$$

The predicted values for these processes in the SM are far below the current sensitivities shown above. 
The projected sensitivities for the rare top decays at the highluminosity LHC (HL LHC) for an integrated luminosity of $3 \mathrm{ab}^{-1}$ at $14 \mathrm{TeV}$ are $\mathcal{O}\left(10^{-4}\right)$ [6,7]. The projections for the Future Circular Collider (FCC) indicate sensitivities comparable to the HL LHC for the $t \rightarrow h u$ decay and about an order of magnitude stronger for the $t \rightarrow h c$ decay [8]. The Compact Linear Collider (CLIC) could also place a limit comparable to the HL LHC for the $t \rightarrow h c$ decay [9].

The Yukawa textures in Sec. II generate flavor-changing couplings for the SM-like Higgs boson, allowing for the rare top decays to appear at tree level. Approximating the total width of the top quark by its dominant partial decay width to a $W$ boson and a $b$ quark, the branching ratios of the rare decays can be written as

$$
\begin{aligned}
& \operatorname{BR}(t \rightarrow h q) \\
& \simeq 2\left|V_{q b}\right|^{2} \frac{\cos ^{2}(\beta-\alpha)}{\sin ^{2} \beta \cos ^{2} \beta} \frac{\left(1-m_{h}^{2} / m_{t, \text { pole }}^{2}\right)^{2}}{\left(1-m_{W}^{2} / m_{t, \text { pole }}^{2}\right)^{2}\left(1+2 m_{W}^{2} / m_{t, \text { pole }}^{2}\right)} \\
& \simeq \frac{\cos ^{2}(\beta-\alpha)}{\sin ^{2} \beta \cos ^{2} \beta} \times\left\{\begin{array}{l}
9.2 \times 10^{-4} \text { for } t \rightarrow h c, \\
8.0 \times 10^{-6} \text { for } t \rightarrow h u .
\end{array}\right.
\end{aligned}
$$

As long as $\cos (\beta-\alpha) \neq 0$, the rare top decay branching ratios can be many orders of magnitude larger than the SM values, making these processes in our model accessible to current and future colliders. If $\cos (\beta-\alpha)=0$ (the socalled alignment limit) the couplings of the $125 \mathrm{GeV}$ Higgs are exactly SM-like. Deviations of $\cos (\beta-\alpha)$ from 0 are constrained by measurements of Higgs production and decays at the LHC. The constraints depend strongly on $\tan \beta$. In Appendix D we show the allowed regions in the $\cos (\beta-\alpha)$ vs $\tan \beta$ plane, taking into account all relevant LHC results on Higgs signal strength measurements.

In Fig. 2 we use these allowed regions to give predictions for the rare top branching ratios as a function of $\tan \beta$ in the type IB model (left) and lepton-specific B model (right). The region in gray is excluded by the current ATLAS limits, while the dotted horizontal lines correspond to projected sensitives from the HL LHC [7], the FCC [8], and CLIC [9].

In the lepton-specific B model, we observe two disjoint regions of parameter space. The upper region opens up for $\tan \beta \gtrsim 10$ and corresponds to a scenario where some of the Higgs couplings differ from the SM prediction by a sign, but are otherwise equal in magnitude. Such a scenario predicts $\operatorname{BR}(t \rightarrow h u) \simeq 6 \times 10^{-5}$ and $\operatorname{BR}(t \rightarrow h c) \simeq 7 \times 10^{-3}$ and is already excluded by the existing LHC constraints from [4].

In general our models can give values for $\operatorname{BR}(t \rightarrow h u)$ and $\operatorname{BR}(t \rightarrow h c)$ that are much larger than the SM prediction, and can be in reach of current or future experimental sensitivities. In the case of $t \rightarrow h u$, the current LHC constraint from [4] does not probe the parameter space of our model. Also future projections from the HL LHC are unlikely to probe our model as they barely touch the region of predicted branching ratio values. The FCC-hh will start to cut into interesting parameter space of $t \rightarrow h u$ with a projected sensitivity of the order of $10^{-5}$.

For the $t \rightarrow h c$ decay channel, the current LHC constraints already probe part of our model parameter space for moderate to large values of $\tan \beta \gtrsim 10$. Projections from the HL LHC and CLIC will be also sensitive to parameter space with much lower choices of $\tan \beta$.

For completeness we also considered the effects of flavor-violating Higgs couplings on the rare top quark decays $t \rightarrow q Z$ and $t \rightarrow q \gamma$, which can be generated at the loop level by closing the Higgs line in the $t \rightarrow h q$ diagram. The branching ratios for these processes can be estimated as

$$
\begin{aligned}
\mathrm{BR}(t \rightarrow q Z / \gamma) & \sim \mathrm{BR}(t \rightarrow h q) \times\left(\frac{g^{2}}{16 \pi^{2}} \frac{m_{t}}{m_{W}}\right)^{2} \\
& \sim \operatorname{BR}(t \rightarrow h q) \times 10^{-5} .
\end{aligned}
$$

The strongest projected sensitivities on $t \rightarrow q Z$ are from FCC-eh [8] and are of the order of $10^{-6}$, while the strongest projected sensitivities on $t \rightarrow q \gamma$ come from FCC-hh [8] and are of the order of $10^{-7}$. Given that the current bounds on $\operatorname{BR}(t \rightarrow h q)$ are $\mathcal{O}\left(10^{-3}\right)$ we predict branching ratios for $t \rightarrow q Z$ and $t \rightarrow q \gamma$ that are at most $\mathcal{O}\left(10^{-8}\right)$, and thus below the sensitivities of current and future experiments, making observation of these processes in our model challenging.

\section{RELATED SIGNATURES}

Although the primary motivation for this model is to explore enhanced $t \rightarrow h q$ decays, the flavor structure of the up Yukawa couplings leads to other interesting features and signatures. We examine possible effects on $D$ meson mixing that arise from tree-level exchange of neutral Higgs bosons. We also consider the collider phenomenology of the heavy neutral and charged Higgs bosons $(H, A$, $H^{ \pm}$), identifying the most prominent production and decay modes.

\section{A. Enhanced D meson mixing from flavorful Higgs bosons}

In the $\mathrm{SM}, D^{0}-\bar{D}^{0}$ mixing proceeds through loop diagrams and is parametrized by the absolute values of the dispersive and absorptive part of the mixing amplitude, $x_{12}=2\left|M_{12}^{D}\right| \tau_{D}, y_{12}=\left|\Gamma_{12}^{D}\right| \tau_{D}$, and their relative phase $\phi_{12}=\operatorname{Arg}\left(M_{12}^{D} / \Gamma_{12}^{D}\right)$, where $\tau_{D}$ is the lifetime of the $D^{0}$ meson.

The current world averages for the mixing parameters are [48]

$$
\begin{aligned}
& x_{12}^{\exp }=\left(0.43_{-0.11}^{+0.10}\right) \%, \quad y_{12}^{\exp }=(0.63 \pm 0.06) \%, \\
& \phi_{12}^{\exp }=\left(-0.25_{-0.99}^{+0.96}\right)^{\circ} .
\end{aligned}
$$



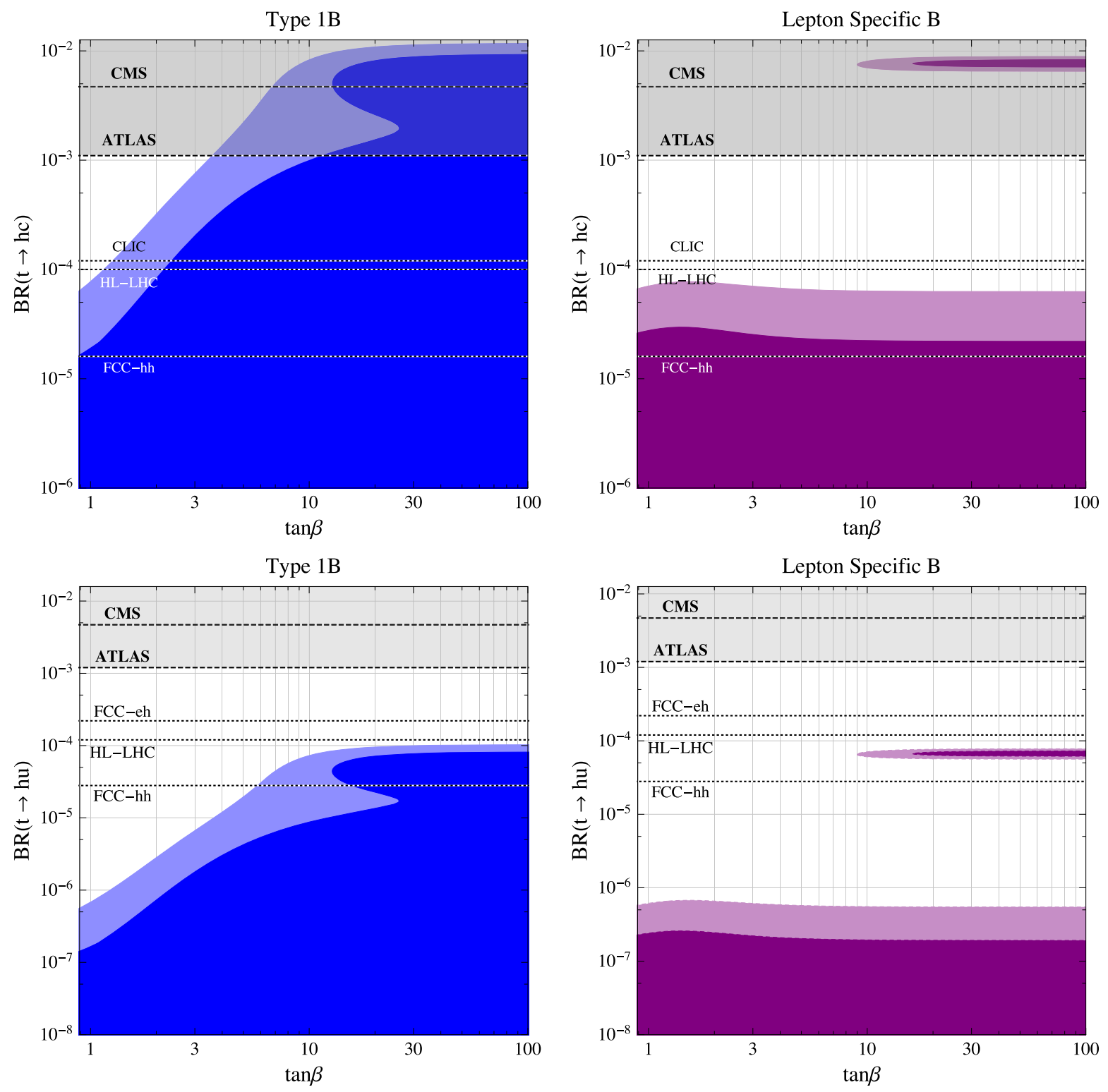

FIG. 2. The branching ratios $t \rightarrow h c$ (top) and $t \rightarrow h u$ (bottom) as a function of $\tan \beta$ in the type IB model (left) and lepton-specific B model (right). The blue and purple shaded regions are consistent with Higgs signal strength measurements. The dashed horizontal lines labeled "ATLAS" are the current best upper bounds on the branching ratios [4]. The dotted horizontal lines are the future projections from the HL LHC, the FCC, and CLIC.

In our model, we generically predict tree-level Higgs contributions to $D^{0}-\bar{D}^{0}$ mixing. The corresponding effect on the dispersive part of the mixing amplitude is given by

$$
\begin{aligned}
M_{12}^{D}= & m_{D}^{3} \frac{f_{D}^{2}}{v_{\mathrm{w}}^{2}} \frac{\left(V_{c b} V_{u b}^{*}\right)^{2}}{s_{\beta}^{2} c_{\beta}^{2}}\left[\frac{1}{4} B_{4} \eta_{4}\left(\frac{c_{\beta-\alpha}^{2}}{m_{h}^{2}}+\frac{s_{\beta-\alpha}^{2}}{m_{H}^{2}}+\frac{1}{m_{A}^{2}}\right) \frac{m_{u}}{m_{c}}\right. \\
& \left.-\left(\frac{5}{24} B_{2} \eta_{2}-\frac{1}{48} B_{3} \eta_{3}\right)\left(\frac{c_{\beta-\alpha}^{2}}{m_{h}^{2}}+\frac{s_{\beta-\alpha}^{2}}{m_{H}^{2}}-\frac{1}{m_{A}^{2}}\right)\right]
\end{aligned}
$$

where the decay constant of the $D^{0}$ meson is $f_{D} \simeq 212 \mathrm{MeV}$ [54], the bag parameters are $B_{2} \simeq 0.65, B_{3} \simeq 0.96, B_{4} \simeq$ 0.91 [55], and the one-loop renormalization group factors are $\eta_{2} \simeq 0.68, \eta_{3} \simeq-0.03, \eta_{4}=1$ [26]. The absorptive part $\Gamma_{12}$ is unaffected in the model. The results for the mixing amplitude are independent of the type of F2HDM; they hold both in type IB and in the lepton-specific B model.

Despite the fact that the neutral Higgs bosons contribute to $D^{0}-\bar{D}^{0}$ mixing at tree level, the approximate $S U(2)^{5}$ flavor symmetry of the F2HDMs ensures that their contributions are very small, suppressed by small quark masses and CKM matrix elements. For Higgs boson masses around $1 \mathrm{TeV}$ and values of $\tan \beta$ as large as 100 , we find that the new physics contribution to $D^{0}-\bar{D}^{0}$ mixing is much smaller than the uncertainties in Eq. (20). Improvements in precision by more than 2 orders of magnitude would be 
required to become sensitive to the predicted nonstandard effects in our models.

\section{B. Collider phenomenology of heavy Higgs bosons}

The F2HDMs considered here offer a rich set of phenomenological consequences. Not only do these models predict additional Higgs bosons that could be within reach of the LHC but the introduction of tree-level FCNCs means that we anticipate distinct signatures coming from the new Higgs bosons that set this model apart from more traditional 2HDMs.

\section{Heavy Higgs production and decays}

There are several production modes via which the heavy Higgs bosons can be produced at the LHC. Due to the enhanced off-diagonal couplings in the up quark sector we expect top associated production, see Fig. 3, to contribute with a sizable cross section.

In order to evaluate the production cross sections, we follow the steps described in $[25,26]$. The results for the production cross sections are identical for the type IB and lepton-specific B models. The cross sections of the heavy neutral Higgs $H$ and the charged Higgs $H^{ \pm}$at $13 \mathrm{TeV}$ proton-proton collisions as a function of $\tan \beta$ for fixed Higgs masses of $1 \mathrm{TeV}$ and $\cos (\beta-\alpha)=0.05$ are shown in the upper plots of Fig. 4. Small values of $\cos (\beta-\alpha) \ll 1$ are motivated by the constraints from Higgs signal strength measurements (see Appendix D).

For the neutral scalar $H$, associated production with a top and gluon-gluon fusion are the dominant production modes. At low $\tan \beta$ gluon-gluon fusion is largest because the coupling to tops is unsuppressed. As $\tan \beta$ increases the gluon-gluon fusion rate drops and is overtaken by top associated production which is enhanced for large $\tan \beta$. The dominant production cross sections for the heavy pseudoscalar $A$ are almost identical to those of the heavy Higgs. For the charged Higgs, top associated production is the largest production mechanism over the full range of $\tan \beta$ values.

The branching ratios of the heavy neutral Higgs $H$ and the charged Higgs $H^{ \pm}$are shown in the lower plots Fig. 4 in

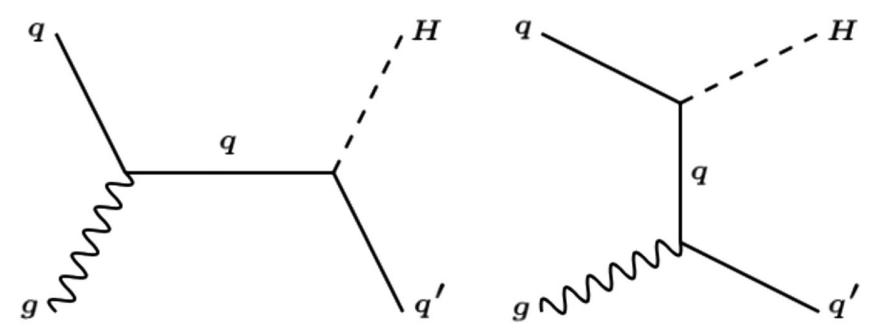

FIG. 3. The Feynman diagrams for quark associated production of the heavy and charged Higgs bosons. In the context of F2HDMs this production mode can have a sizeable cross section due to the tree-level flavor-changing neutral currents. the type IB model. Results in the lepton-specific B model are almost identical. The main difference in the leptonspecific B model is the presence of a $\tau \tau$ branching ratio at the level of a few percent, which is strongly suppressed in the type IB model.

As expected, for moderate to large $\tan \beta$ the dominant decay mode of $H$ is the flavor-changing $H \rightarrow c t$. The branching ratio to $t \bar{t}$ can be substantial; however this decay mode primarily plays a role for small $\tan \beta$ which is less motivated by the quark mass hierarchy. For moderate $\tan \beta$ we also notice that the gauge bosons can contribute at a level between $1 \%$ and $10 \%$. The branching ratios of the charged Higgs tend to be dominated by $t s$ and $t b$ decays. In particular, for low $\tan \beta, t b$ dominates. Once $\tan \beta$ becomes larger than about 5 we see $t s$ dominates for the rest of the parameter space. In addition to the most dominant decays, we see that at the level of a few percent or lower we can expect decays to $W^{ \pm} h$.

\section{Collider signatures}

The constraints on this model from existing searches for the heavy Higgs boson are very weak due to the unique flavor structure. The overwhelming decay of the neutral Higgses to $c t$ means the branching ratio to other modes is highly suppressed as seen in Fig. 4. Typical search channels at the LHC are through these suppressed channels, such as $\mu \mu, \tau \tau, V V$, and $j j$, making the prospects of discovering a heavy Higgs through these channels very weak. Also, the standard searches for charged Higgs bosons in the $\tau \nu$ channel hardly constrain our parameter space, due to the strongly reduced branching ratios $H^{ \pm} \rightarrow \tau \nu$. Unique signatures that are relevant to collider searches of our model are driven by the large nonstandard decay modes $H, A \rightarrow t c$, and $H^{ \pm} \rightarrow t s$.

The charged Higgs produced via top associated production and subsequent decay to $t s$ leads to opposite-sign tops that do not reconstruct a resonance. This is similar to charged Higgs bosons in 2HDMs with natural flavor conservation. The unique feature with respect to $2 \mathrm{HDMs}$ with natural flavor conservation is that the accompanying jet of the $t \bar{t}$ system is not a $b$ jet but a strange jet. The cross section for the $t \bar{t}+$ jet signature as a function of $\tan \beta$ and charged Higgs mass is shown in the right plot of Fig. 5. We find cross sections that can easily exceed $100 \mathrm{fb}$ for Higgs masses of $O(1 \mathrm{TeV})$ and sizable $\tan \beta$. The shaded region to the left of the vertical line at Higgs masses of around $800 \mathrm{GeV}$ is excluded by the constraint from $b \rightarrow s \gamma$. Existing searches for charged Higgs bosons that decay to $t b[56,57]$ make heavy use of $\mathrm{b}$ tagging and are therefore not directly applicable in our scenario. Our work motivates dedicated studies of the $p p \rightarrow t H^{-} \rightarrow t \bar{t} s$ signature.

The heavy neutral Higgs being produced through top associated production along with a decay into tc leads also to a final state with ditops that do not reconstruct a 

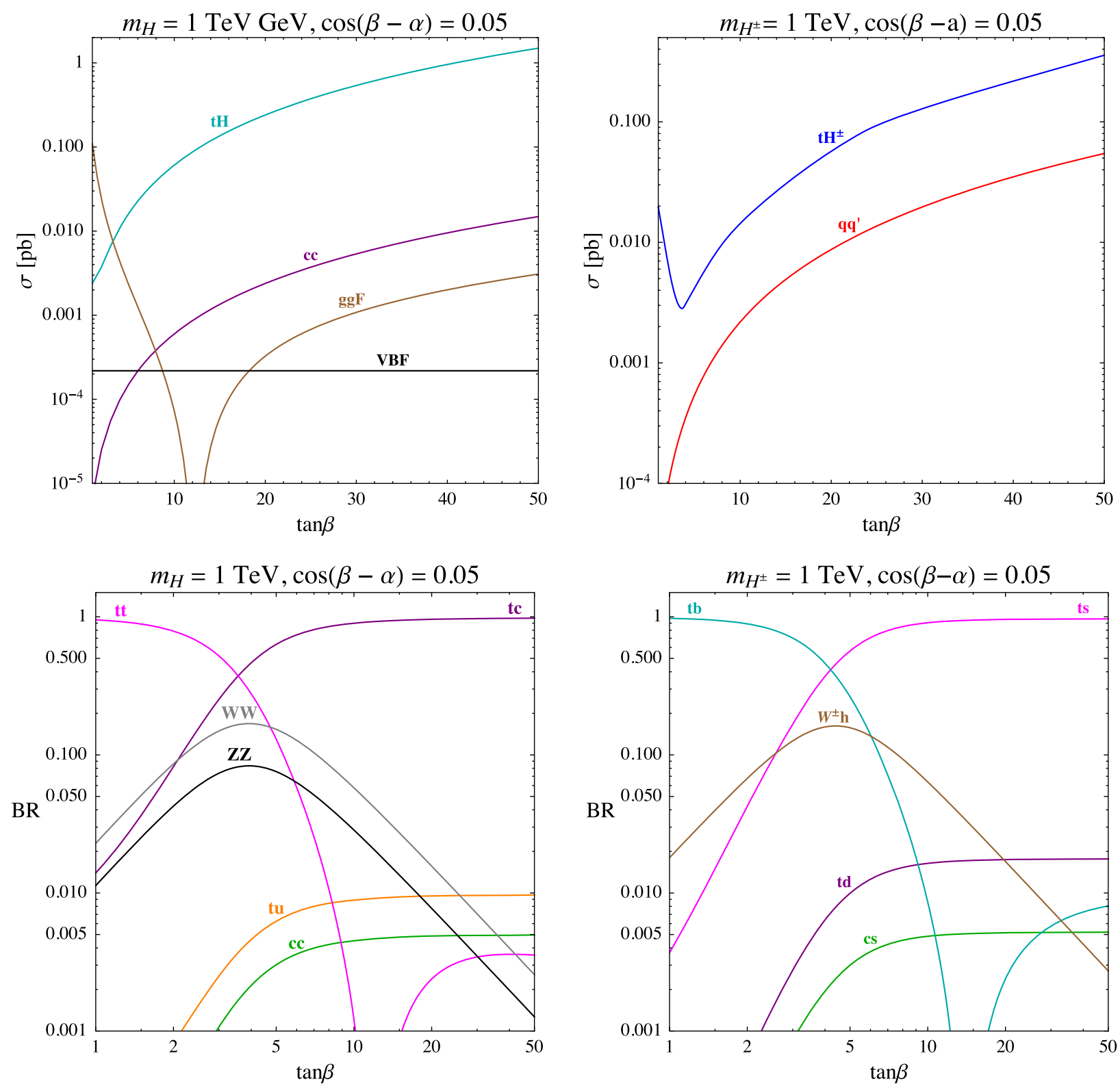

FIG. 4. Production cross sections (top) and branching ratios (bottom) of the heavy neutral Higgs $H$ (left) and the charged Higgs $H^{ \pm}$(right) in the flavorful 2HDM of type IB as a function of $\tan \beta$ with the masses $m_{H}$ and $m_{H^{ \pm}}$fixed to 1 TeV and $\cos (\beta-\alpha)=0.05$.

resonance. In our flavorful $2 \mathrm{HDMs}, 50 \%$ of the time the final state will be same-sign tops, in contrast to $2 \mathrm{HDMs}$ with natural flavor conservation that only produce opposite-sign tops. Same-sign tops have been identified as important probes in a number of new physics scenarios, including R-parity violating supersymmetry [59], 2HDMs $[37,60,61]$, additional scalars [62,63], colored vectors [64], and effective field theories [65]. The cross section of samesign tops in our scenario is shown in the left plot of Fig. 5 in the plane of Higgs mass vs $\tan \beta$. For Higgs masses of $O(1 \mathrm{TeV})$ we find cross sections up to $1 \mathrm{pb}$. The shaded region to the left of the vertical line at Higgs masses of around $800 \mathrm{GeV}$ is excluded by the constraint from $b \rightarrow s \gamma$ assuming that $m_{H} \simeq m_{H^{ \pm}}$.

In [58] searches for same-sign leptons are interpreted in a benchmark model in which same-sign tops are created by a neutral spin-1 mediator. Assuming that the acceptances and efficiencies are comparable in our scenario with a scalar mediator, we find that the large $\tan \beta$ region is already partly probed by the existing search. We show the region that is excluded by the same-sign top search also in the charged Higgs plot, assuming that $m_{H} \simeq m_{H^{ \pm}}$. Keeping in mind that our $p p \rightarrow H t \rightarrow t t \bar{c}$ cross section approximately scales $\operatorname{as}^{2} \tan ^{2} \beta$, we expect that parameter space with $\tan \beta$ 

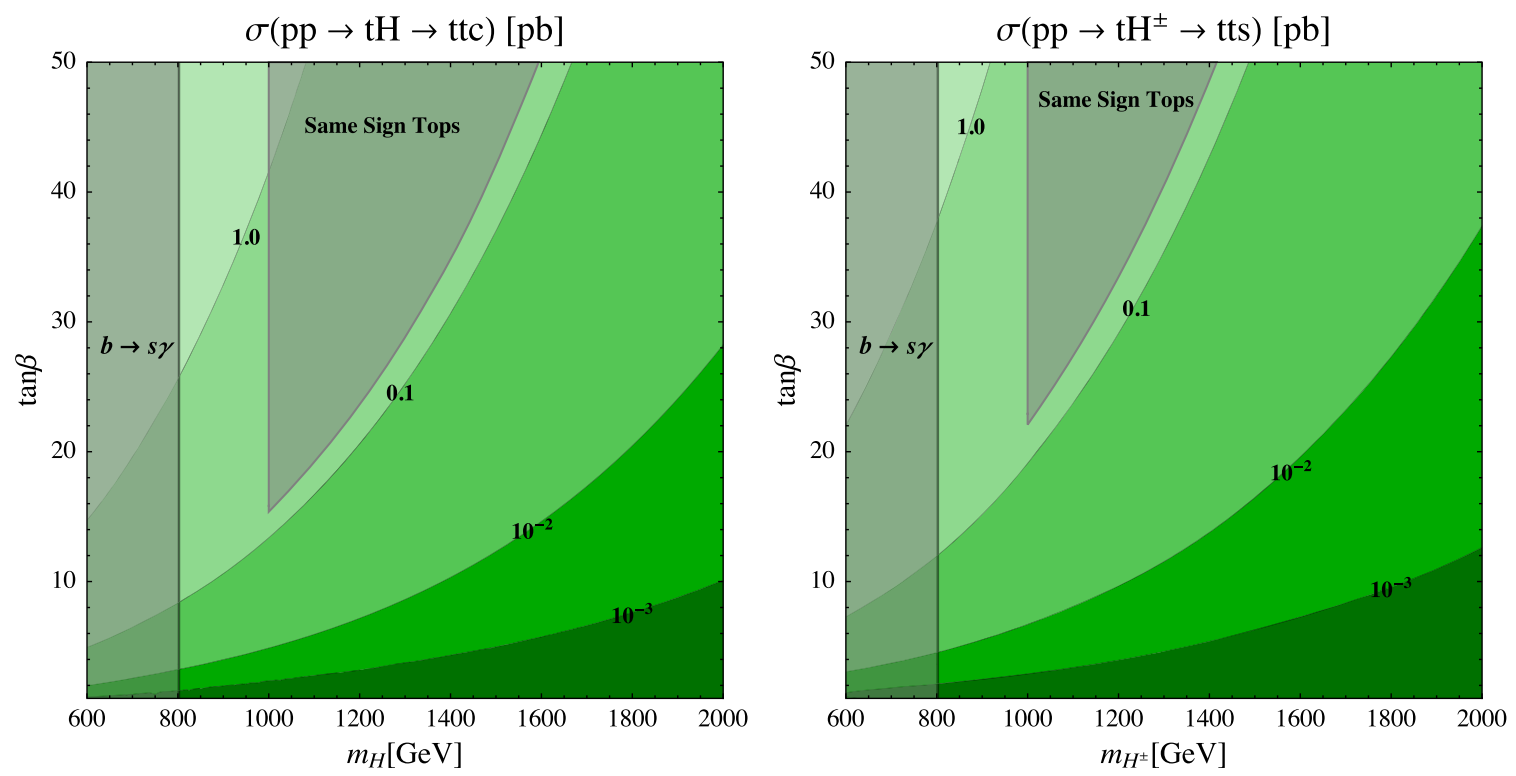

FIG. 5. Cross section of same-sign tops plus jet from the production and decay of a neutral heavy Higgs $H$ (left) as well as oppositesign tops plus jet from the production and decay of a charged Higgs (right) in the considered flavorful 2HDM of type IB in the plane of Higgs mass vs $\tan \beta$. The gray shaded regions for light Higgs masses are excluded by $b \rightarrow s \gamma$ constraints (see Sec. III). The triangle-shaped gray region for large $\tan \beta$ is excluded by existing searches for same-sign tops [58]. Throughout the plots we set $\cos (\beta-\alpha)=0.05$.

as low as $\sim 10$ might be probed by same-sign top searches at the high-luminosity LHC.

\section{CONCLUSIONS}

Rare top decays are strongly suppressed in the SM and their observation at existing or planned colliders would be a clear indication of new physics. One new physics framework that can lead to branching ratios of $t \rightarrow h c$ and $t \rightarrow h u$ in reach of current or future colliders is flavorful 2HDMs.

In this work we explored a version of flavorful 2HDM where quark mixing dominantly resides in the up quark sector, leading to FCNCs in the up quark sector at tree level. We constructed a flavor model based on $U(1)$ flavor symmetries which successfully reproduces the measured quark masses and CKM mixing angles. We found that our model is highly predictive as the flavor structure of all Higgs couplings is fully determined by the quark masses and CKM matrix elements.

We gave predictions for $t \rightarrow h c$ and $t \rightarrow h u$ rates in our model and showed that the branching ratios can reach values of $\operatorname{BR}(t \rightarrow h c) \sim 10^{-2}$ and $\operatorname{BR}(t \rightarrow h u) \sim 10^{-4}$ (see Fig. 2) without violating constraints from Higgs signal strength measurements at the LHC. Existing bounds on $\operatorname{BR}(t \rightarrow h c)$ from the LHC already start to constrain model parameter space. Expected sensitivities at the highluminosity LHC or future colliders will be able to probe broad regions of parameter space. In passing we also provided updated predictions for the $t \rightarrow h c$ and $t \rightarrow h u$ branching ratios in the SM [see Eq. (15)].
We explored additional effects of the up quark FCNCs in low energy flavor-violating processes. In particular, we found that one-loop effects in the rare $B$ decay $b \rightarrow s \gamma$ lead to strong constraints on the masses of the additional Higgs bosons of at least $\sim 800 \mathrm{GeV}$. On the other hand, constraints from $D$ meson mixing are weak in our setup.

Finally, we explored the phenomenology of the heavy neutral and charged Higgs bosons of the F2HDM. We found that both neutral and charged Higgses are mainly produced in association with top quarks. The by far dominant decay modes are $t c$ and $t s$, respectively. These final states are not typical search channels of Higgs bosons in traditional 2HDMs. Therefore, current constraints from colliders are weak. The most prominent signatures of the models are $p p \rightarrow t H^{-} \rightarrow t \bar{t} s$, i.e., opposite-sign tops + jet, and in particular $p p \rightarrow t H \rightarrow t t \bar{c}$, i.e., same-sign tops + jet. Cross sections of these signatures can be of the order of $100 \mathrm{fb}$ for Higgs masses around $1 \mathrm{TeV}$ (see Fig. 5). Our results in the F2HDM framework motivate continued searches for same-sign tops and provide an additional benchmark model in which future same-sign top searches can be interpreted.

\section{ACKNOWLEDGMENTS}

We thank Stefania Gori for useful discussions. The research of W. A. is supported by the National Science Foundation under Grant No. PHY-1912719. 


\section{APPENDIX A: YUKAWA COUPLINGS IN THE QUARK MASS EIGENSTATE BASIS}

In this Appendix we show that in the considered type IB and lepton-specific B models, the couplings of the Higgs bosons to the quarks in the quark mass eigenstate basis are entirely determined by the known quark masses and CKM elements.

The starting point is the Yukawa couplings $\lambda^{q}$ and $\lambda^{\prime q}$ in Eq. (1) that need to be rotated into the quark mass eigenstate basis. We perform unitary rotations on the left-handed and right-handed quark fields $q_{L / R} \rightarrow U_{q_{L / R}} q_{L / R}$ such that $U_{u_{L}}^{\dagger}\left(v \lambda^{u}+v^{\prime} \lambda^{\prime u}\right) U_{u_{R}}=\operatorname{diag}\left(m_{u}, m_{c}, m_{t}\right) \equiv m_{u}^{\text {diag }}$ and analogous for the down quarks. Given the structure of $\lambda^{u}$ and $\lambda^{\prime u}$ in Eq. (4a) we can introduce the matrix $\Pi=\operatorname{diag}(0,0,1)$ that leaves $\lambda^{u}$ invariant and that annihilates $\lambda^{\prime u}: \Pi \lambda^{u}=\lambda^{u}$ and $\Pi \lambda^{\prime u}=0$. Using this matrix, we can express $\lambda^{u}$ in the mass eigenstate basis directly in terms of quark masses and the CKM matrix

$$
\begin{aligned}
U_{u_{L}}^{\dagger} \lambda^{u} U_{u_{R}} & =U_{u_{L}}^{\dagger} \Pi\left(\lambda^{u}+\frac{v^{\prime}}{v} \lambda^{\prime u}\right) U_{u_{R}}=\frac{\sqrt{2}}{v} U_{u_{L}}^{\dagger} \Pi U_{u_{L}} m_{u}^{\text {diag }} \\
& =\frac{\sqrt{2}}{v} V_{\mathrm{CKM}} \Pi V_{\mathrm{CKM}}^{\dagger} m_{u}^{\text {diag }} .
\end{aligned}
$$

In the last step we used the definition of the CKM matrix $V_{\mathrm{CKM}}=U_{u_{L}}^{\dagger} U_{d_{L}}$ and the fact that $U_{d_{L}}$ and $\Pi$ commute due to the structure of $\lambda^{d}$ and $\lambda^{\prime d}$ in Eq. (4b). Analogously, we can use the matrix $\Pi^{\prime}=\operatorname{diag}(1,1,0)$ to get an expression for $\lambda^{\prime u}$ in the mass eigenstate basis

$$
\begin{aligned}
U_{u_{L}}^{\dagger} \lambda^{\prime u} U_{u_{R}} & =U_{u_{L}}^{\dagger} \Pi^{\prime}\left(\frac{v}{v^{\prime}} \lambda^{u}+\lambda^{\prime u}\right) U_{u_{R}} \\
& =\frac{\sqrt{2}}{v^{\prime}} U_{u_{L}}^{\dagger} \Pi^{\prime} U_{u_{L}} m_{u}^{\text {diag }} \\
& =\frac{\sqrt{2}}{v^{\prime}} V_{\mathrm{CKM}} \Pi^{\prime} V_{\mathrm{CKM}}^{\dagger} m_{u}^{\text {diag }} .
\end{aligned}
$$

In the down quark sector we instead get

$$
\begin{aligned}
U_{d_{L}}^{\dagger} \lambda^{d} U_{d_{R}} & =U_{d_{L}}^{\dagger} \Pi\left(\lambda^{d}+\frac{v^{\prime}}{v} \lambda^{\prime d}\right) U_{d_{R}}=\frac{\sqrt{2}}{v} U_{d_{L}}^{\dagger} \Pi U_{d_{L}} m_{d}^{\text {diag }} \\
& =\frac{\sqrt{2}}{v} \Pi m_{d}^{\text {diag }}
\end{aligned}
$$

$$
\begin{aligned}
U_{d_{L}}^{\dagger} \lambda^{\prime d} U_{d_{R}} & =U_{d_{L}}^{\dagger} \Pi^{\prime}\left(\frac{v}{v^{\prime}} \lambda^{d}+\lambda^{\prime d}\right) U_{d_{R}} \\
& =\frac{\sqrt{2} 1}{v^{\prime}} U_{d_{L}}^{\dagger} \Pi^{\prime} U_{d_{L}} m_{d}^{\text {diag }}=\frac{\sqrt{2}}{v^{\prime}} \Pi^{\prime} m_{d}^{\text {diag }} .
\end{aligned}
$$

\section{APPENDIX B: LOOP FUNCTIONS FOR $b \rightarrow s \gamma$}

In this Appendix we give the explicit expressions for the loop functions that enter the results for the charged Higgs contributions to the $b \rightarrow s \gamma$ decay in Sec. III:

$$
\begin{aligned}
f_{7}(x) & =\frac{(2-3 x)^{2} \log x}{12(1-x)^{4}}+\frac{11-43 x+38 x^{2}}{72(1-x)^{3}}, \\
\lim _{x \rightarrow 0} f_{7}(x) & =\frac{1}{3} \log (x)+\frac{11}{72}, \\
f_{8}(x) & =\frac{(2-3 x) \log x}{4(1-x)^{4}}+\frac{16-29 x+7 x^{2}}{24(1-x)^{3}}, \\
\lim _{x \rightarrow 0} f_{8}(x) & =\frac{1}{2} \log (x)+\frac{2}{3}, \\
g_{7}(x) & =-\frac{x(2-3 x) \log x}{12(1-x)^{4}}-\frac{7-5 x-8 x^{2}}{72(1-x)^{3}}, \\
\lim _{x \rightarrow 0} g_{7}(x) & =-\frac{7}{72}, \\
g_{8}(x) & =-\frac{x \log x}{4(1-x)^{4}}-\frac{2+5 x-x^{2}}{24(1-x)^{3}}, \quad \lim _{x \rightarrow 0} g_{8}(x)=-\frac{1}{12}, \\
h_{7}(x) & =\frac{(2-3 x) \log x}{6(1-x)^{3}}+\frac{3-5 x}{12(1-x)^{2}}, \\
\lim _{x \rightarrow 0} h_{7}(x) & =\frac{1}{3} \log (x)+\frac{1}{4}, \\
h_{8}(x) & =\frac{\log x}{2(1-x)^{3}+\frac{3}{4(1-x)^{2}}, \quad \lim _{x \rightarrow 0} h_{8}(x)=\frac{1}{2} \log (x)+\frac{3}{4} .}
\end{aligned}
$$

\section{APPENDIX C: LOOP FUNCTION FOR $\boldsymbol{t} \rightarrow \boldsymbol{h} q$}

The loop function $\mathcal{F}$ that enters our SM expression of the rare top branching ratios Eq. (15) can be written as

$$
\begin{aligned}
\mathcal{F}(x, y)= & \frac{1}{16(x-y)^{2}} \mid x\left(B_{0}(0,0,1)-B_{0}(x, 0,1)\right)+y\left(B_{0}(y, 0,0)-B_{0}(0,0,1)\right) 4\left(B_{0}(x, 0,1)-B_{0}(y, 0,0)\right) \\
& +(2+y)\left(B_{0}(y, 1,1)-B_{0}(x, 1,0)\right)-(4-2 x+y) C_{0}(y, x, 0,0,0,1)+\left(2-y+y^{2}-x(2+y)\right) C_{0}(y, x, 0,1,1,0) \\
& +2(y-x) B_{0}^{\prime}(0,1,0)+2(x-2) B_{0}^{\prime}(x, 1,0)+\left.2\left(x^{2}+y-x y-2\right) C_{0}^{\prime}(y, x, 0,1,1,0)\right|^{2},
\end{aligned}
$$


with the following definitions of the Passarino-Veltman functions

$$
\begin{aligned}
& \frac{i}{16 \pi^{2}} B_{0}\left(p^{2}, m_{1}^{2}, m_{2}^{2}\right) \\
& \quad=\bar{\mu}^{4-D} \int \frac{\mathrm{d}^{D} q}{(2 \pi)^{D}} \frac{1}{\left(q^{2}-m_{1}^{2}\right)\left((q+p)^{2}-m_{2}^{2}\right)}, \quad(\mathrm{C} 2) \\
& \frac{i}{16 \pi^{2}} C_{0}\left(p^{2}, k^{2},(p+k)^{2}, m_{1}^{2}, m_{2}^{2}, m_{3}^{2}\right) \\
& =\int \frac{\mathrm{d}^{4} q}{(2 \pi)^{4}} \frac{1}{\left(q^{2}-m_{1}^{2}\right)\left((q+p)^{2}-m_{2}^{2}\right)\left((q+p+k)^{2}-m_{3}^{2}\right)} .
\end{aligned}
$$

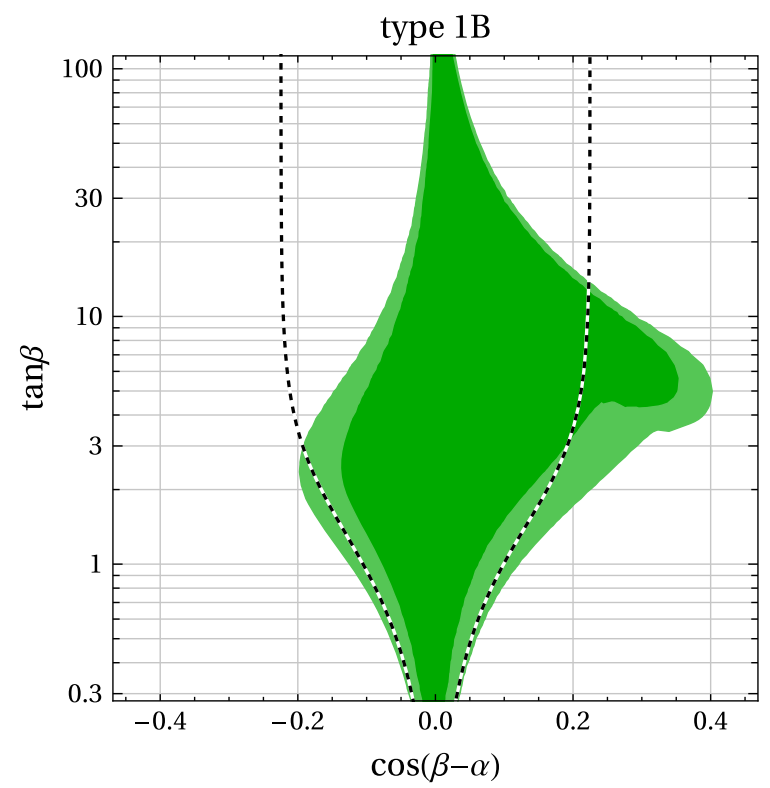

lepton-specific B

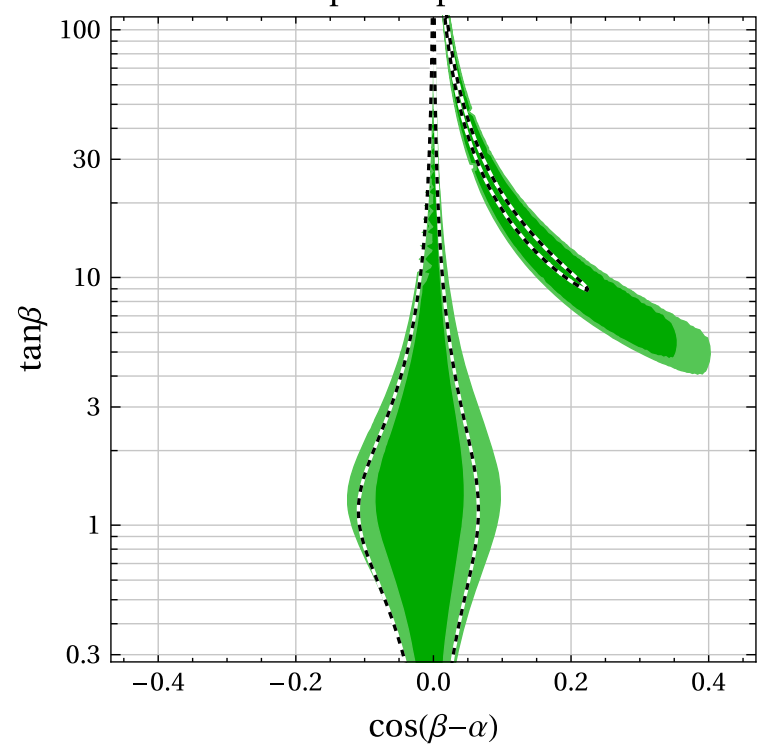

The derivatives in Eq. (C1) act on the last argument of the functions, i.e.,

$$
\begin{gathered}
B_{0}^{\prime}(a, b, c)=\frac{\partial}{\partial c} B_{0}(a, b, c), \\
C_{0}^{\prime}(a, b, c, d, e, f)=\frac{\partial}{\partial f} C_{0}(a, b, c, d, e, f) .
\end{gathered}
$$

\section{APPENDIX D: HIGGS SIGNAL STRENGTH FIT}

Away from the alignment limit $\cos (\beta-\alpha)=0$, the couplings of the $125 \mathrm{GeV}$ Higgs boson differ from their SM predictions. Therefore, signal strength measurements
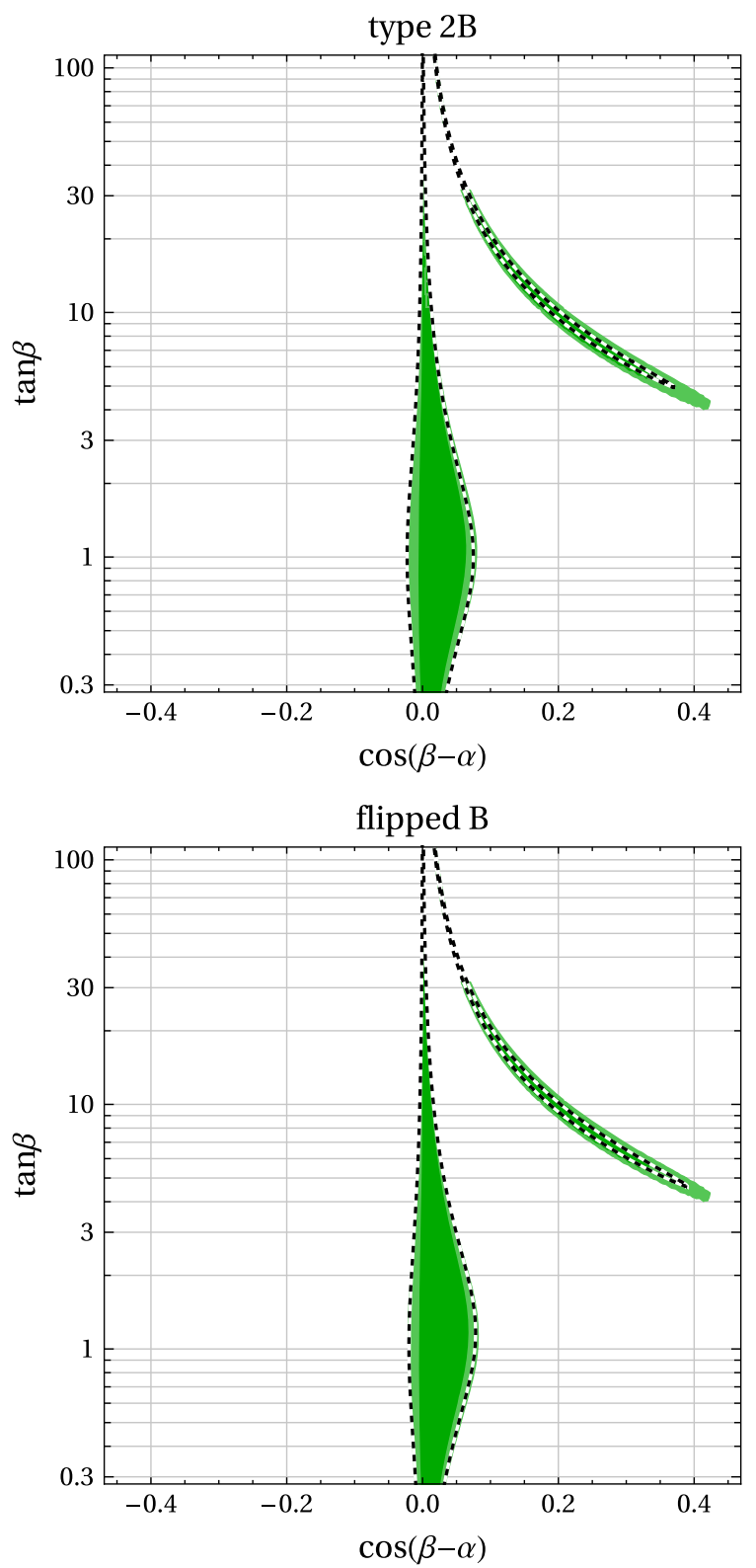

FIG. 6. Constraints in the $\cos (\beta-\alpha)$ vs $\tan \beta$ plane based on LHC measurements of the $125 \mathrm{GeV}$ Higgs signal strengths. Parameter space of the flavorful $2 \mathrm{HDMs}$ that is compatible with the data at the $1 \sigma$ and $2 \sigma$ level is shown in green. For comparison, the $2 \sigma$ regions in the corresponding $2 \mathrm{HDMs}$ with natural flavor conservation are shown by dashed contours. 
from ATLAS and CMS can be used to constrain the parameter space of our 2HDMs. With respect to our previous signal strength analysis in [26], we include LHC Run 2 updates of $h \rightarrow W W$ [66-68], $h \rightarrow \tau \tau$ [11,69], $h \rightarrow \mu \mu$ [16], the recent $h \rightarrow b b$ observations [12,13], and the results for $t$ th production $[14,15]$.
In Fig. 6 we show the allowed ranges in the $\cos (\beta-\alpha)$ vs $\tan \beta$ plane in the type IB model (top left), type IIB model (top right), lepton-specific $\mathrm{B}$ model (bottom left), and flipped B model (bottom right) at $1 \sigma$ (dark green) and $2 \sigma$ (light green). The dotted lines indicate the $2 \sigma$ constraint in the corresponding $2 \mathrm{HDMs}$ with natural flavor conservation.
[1] S. L. Glashow, J. Iliopoulos, and L. Maiani, Weak interactions with lepton-hadron symmetry, Phys. Rev. D 2, 1285 (1970).

[2] B. Mele, S. Petrarca, and A. Soddu, A new evaluation of the $t \rightarrow c H$ decay width in the standard model, Phys. Lett. B 435, 401 (1998).

[3] J. A. Aguilar-Saavedra, Top flavor-changing neutral interactions: Theoretical expectations and experimental detection, Acta Phys. Pol. B 35, 2695 (2004).

[4] M. Aaboud et al. (ATLAS Collaboration), Search for topquark decays $t \rightarrow H q$ with $36 \mathrm{fb}^{-1}$ of $p p$ collision data at $\sqrt{s}=13 \mathrm{TeV}$ with the ATLAS detector, J. High Energy Phys. 05 (2019) 123.

[5] A. M. Sirunyan et al. (CMS Collaboration), Search for the flavor-changing neutral current interactions of the top quark and the Higgs boson which decays into a pair of $b$ quarks at $\sqrt{s}=13 \mathrm{TeV}$, J. High Energy Phys. 06 (2018) 102.

[6] ATLAS Collaboration, Expected sensitivity of ATLAS to FCNC top quark decays $t \rightarrow \mathrm{Zu}$ and $t \rightarrow H q$ at the high luminosity LHC, Report No. ATL-PHYS-PUB-2016-019, 2016.

[7] A. Cerri et al., Opportunities in flavour physics at the HL-LHC and HE-LHC, arXiv:1812.07638.

[8] M. Mangano et al., Future circular collider: Vol. 1 Physics opportunities, Report No. CERN-ACC-2018-0056, 2018.

[9] J. de Blas et al., The CLIC potential for new physics, CERN Yellow Rep. Monogr. 3 (2018).

[10] A. M. Sirunyan et al. (CMS Collaboration), Observation of the Higgs boson decay to a pair of $\tau$ leptons with the CMS detector, Phys. Lett. B 779, 283 (2018).

[11] M. Aaboud et al. (ATLAS Collaboration), Cross-section measurements of the Higgs boson decaying into a pair of $\tau$-leptons in proton-proton collisions at $\sqrt{s}=13 \mathrm{TeV}$ with the ATLAS detector, Phys. Rev. D 99, 072001 (2019).

[12] A. M. Sirunyan et al. (CMS Collaboration), Observation of Higgs Boson Decay to Bottom Quarks, Phys. Rev. Lett. 121, 121801 (2018).

[13] M. Aaboud et al. (ATLAS Collaboration), Observation of $H \rightarrow b \bar{b}$ decays and $V H$ production with the ATLAS detector, Phys. Lett. B 786, 59 (2018).

[14] A. M. Sirunyan et al. (CMS Collaboration), Observation of $t \bar{t} H$ Production, Phys. Rev. Lett. 120, 231801 (2018).

[15] M. Aaboud et al. (ATLAS Collaboration), Observation of Higgs boson production in association with a top quark pair at the LHC with the ATLAS detector, Phys. Lett. B 784, 173 (2018).
[16] A. M. Sirunyan et al. (CMS Collaboration), Search for the Higgs Boson Decaying to Two Muons in Proton-Proton Collisions at $\sqrt{s}=13 \mathrm{TeV}$, Phys. Rev. Lett. 122, 021801 (2019).

[17] M. Aaboud et al. (ATLAS Collaboration), Search for the Dimuon Decay of the Higgs Boson in $p p$ Collisions at $\sqrt{s}=13 \mathrm{TeV}$ with the ATLAS Detector, Phys. Rev. Lett. 119, 051802 (2017).

[18] W. Altmannshofer, S. Gori, A. L. Kagan, L. Silvestrini, and J. Zupan, Uncovering mass generation through Higgs flavor violation, Phys. Rev. D 93, 031301 (2016).

[19] F. J. Botella, G. C. Branco, M. N. Rebelo, and J. I. SilvaMarcos, What if the masses of the first two quark families are not generated by the standard model Higgs boson?, Phys. Rev. D 94, 115031 (2016).

[20] D. Ghosh, R. S. Gupta, and G. Perez, Is the Higgs mechanism of fermion mass generation a fact? A Yukawa-less first-twogeneration model, Phys. Lett. B 755, 504 (2016).

[21] A. K. Das and C. Kao, A two Higgs doublet model for the top quark, Phys. Lett. B 372, 106 (1996).

[22] A. E. Blechman, A. A. Petrov, and G. Yeghiyan, The flavor puzzle in multi-Higgs models, J. High Energy Phys. 11 (2010) 075.

[23] S. Knapen and D. J. Robinson, Disentangling Mass and Mixing Hierarchies, Phys. Rev. Lett. 115, 161803 (2015).

[24] W. Altmannshofer, S. Gori, D. J. Robinson, and D. Tuckler, The flavor-locked flavorful two Higgs doublet model, J. High Energy Phys. 03 (2018) 129.

[25] W. Altmannshofer, J. Eby, S. Gori, M. Lotito, M. Martone, and D. Tuckler, Collider signatures of flavorful Higgs bosons, Phys. Rev. D 94, 115032 (2016).

[26] W. Altmannshofer and B. Maddock, Flavorful two Higgs doublet models with a twist, Phys. Rev. D 98, 075005 (2018).

[27] A. Crivellin, A. Kokulu, and C. Greub, Flavor-phenomenology of two-Higgs-doublet models with generic Yukawa structure, Phys. Rev. D 87, 094031 (2013).

[28] M. Bauer, M. Carena, and K. Gemmler, Flavor from the electroweak scale, J. High Energy Phys. 11 (2015) 016.

[29] C. W. Chiang, H. Fukuda, M. Takeuchi, and T. T. Yanagida, Flavor-changing neutral-current decays in top-specific variant axion model, J. High Energy Phys. 11 (2015) 057.

[30] A. Crivellin, J. Heeck, and P. Stoffer, A Perturbed LeptonSpecific Two-Higgs-Doublet Model Facing Experimental Hints for Physics beyond the Standard Model, Phys. Rev. Lett. 116, 081801 (2016). 
[31] F. J. Botella, G. C. Branco, M. Nebot, and M. N. Rebelo, Flavour changing Higgs couplings in a class of two Higgs doublet models, Eur. Phys. J. C 76, 161 (2016).

[32] M. Bauer, M. Carena, and K. Gemmler, Creating the fermion mass hierarchies with multiple Higgs bosons, Phys. Rev. D 94, 115030 (2016).

[33] M. Buschmann, J. Kopp, J. Liu, and X. P. Wang, New signatures of flavor violating Higgs couplings, J. High Energy Phys. 06 (2016) 149.

[34] M. Sher and K. Thrasher, Flavor changing leptonic decays of heavy Higgs bosons, Phys. Rev. D 93, 055021 (2016).

[35] R. Primulando and P. Uttayarat, Probing lepton flavor violation at the $13 \mathrm{TeV}$ LHC, J. High Energy Phys. 05 (2017) 055.

[36] J. M. Alves, F. J. Botella, G. C. Branco, F. Cornet-Gomez, and M. Nebot, Controlled flavour changing neutral couplings in two Higgs doublet models, Eur. Phys. J. C 77, 585 (2017).

[37] S. Gori, C. Grojean, A. Juste, and A. Paul, Heavy Higgs searches: Flavour matters, J. High Energy Phys. 01 (2018) 108.

[38] A. Crivellin, J. Heeck, and D. Müller, Large $h \rightarrow b s$ in generic two-Higgs-doublet models, Phys. Rev. D 97, 035008 (2018).

[39] M. Kohda, T. Modak, and W. S. Hou, Searching for new scalar bosons via triple-top signature in $c g \rightarrow t S^{0} \rightarrow t t \bar{t}$, Phys. Lett. B 776, 379 (2018).

[40] M. Badziak and K. Harigaya, Asymptotically Free Natural Supersymmetric Twin Higgs Model, Phys. Rev. Lett. 120, 211803 (2018).

[41] A. Dery, C. Frugiuele, and Y. Nir, Large Higgs-electron Yukawa coupling in 2HDM, J. High Energy Phys. 04 (2018) 044.

[42] S. Banerjee, M. Chala, and M. Spannowsky, Top quark FCNCs in extended Higgs sectors, Eur. Phys. J. C 78, 683 (2018).

[43] J. L. Díaz-Cruz, B. O. Larios-Lopez, and M. A. Perez-de Leon, A private SUSY 4HDM with FCNC in the up-sector, arXiv: 1901.01304.

[44] P. M. Ferreira and L. Lavoura, No strong $C P$ violation up to the one-loop level in a two-Higgs-doublet model, arXiv: 1904.08438 .

[45] C. D. Froggatt and H. B. Nielsen, Hierarchy of quark masses, Cabibbo angles and $C P$ violation, Nucl. Phys. B147, 277 (1979).

[46] S. L. Glashow and S. Weinberg, Natural conservation laws for neutral currents, Phys. Rev. D 15, 1958 (1977).

[47] M. Misiak et al., Updated NNLO QCD Predictions for the Weak Radiative B-Meson Decays, Phys. Rev. Lett. 114, 221801 (2015).

[48] Y. Amhis et al. (HFLAV Collaboration), Averages of $b$-hadron, $c$-hadron, and $\tau$-lepton properties as of summer 2016, Eur. Phys. J. C 77, 895 (2017).

[49] C. Bobeth, M. Misiak, and J. Urban, Matching conditions for $b \rightarrow s \gamma$ and $b \rightarrow s$ gluon in extensions of the standard model, Nucl. Phys. B567, 153 (2000).

[50] A. Paul and D. M. Straub, Constraints on new physics from radiative $B$ decays, J. High Energy Phys. 04 (2017) 027.
[51] G. Eilam, B. Haeri, and A. Soni, Flavor changing Higgs transitions, Phys. Rev. D 41, 875 (1990).

[52] G. Abbas, A. Celis, X. Q. Li, J. Lu, and A. Pich, Flavourchanging top decays in the aligned two-Higgs-doublet model, J. High Energy Phys. 06 (2015) 005.

[53] M. Tanabashi et al. (Particle Data Group), Review of particle physics, Phys. Rev. D 98, 030001 (2018).

[54] S. Aoki et al. (Flavour Lattice Averaging Group), FLAG review 2019, arXiv:1902.08191.

[55] N. Carrasco, P. Dimopoulos, R. Frezzotti, V. Lubicz, G. C. Rossi, S. Simula, and C. Tarantino (ETM Collaboration), $\Delta S=2$ and $\Delta C=2$ bag parameters in the standard model and beyond from $\mathrm{N}_{f}=2+1+1$ twisted-mass lattice QCD, Phys. Rev. D 92, 034516 (2015).

[56] M. Aaboud et al. (ATLAS Collaboration), Search for charged Higgs bosons decaying into top and bottom quarks at $\sqrt{s}=13 \mathrm{TeV}$ with the ATLAS detector, J. High Energy Phys. 11 (2018) 085.

[57] CMS Collaboration, Search for a charged Higgs boson decaying into top and bottom quarks in proton-proton collisions at $13 \mathrm{TeV}$ in events with electrons or muons, Report No. CMS-PAS-HIG-18-004, 2018.

[58] M. Aaboud et al. (ATLAS Collaboration), Search for new phenomena in events with same-charge leptons and $b$-jets in $p p$ collisions at $\sqrt{s}=13 \mathrm{TeV}$ with the ATLAS detector, J. High Energy Phys. 12 (2018) 039.

[59] G. Durieux and C. Smith, The same-sign top signature of $R$-parity violation, J. High Energy Phys. 10 (2013) 068.

[60] C. S. Kim, Y. W. Yoon, and X. B. Yuan, Exploring top quark FCNC within 2HDM type III in association with flavor physics, J. High Energy Phys. 12 (2015) 038.

[61] W. S. Hou, M. Kohda, and T. Modak, Constraining a lighter exotic scalar via same-sign top, Phys. Lett. B 786, 212 (2018).

[62] S. Bar-Shalom, A. Rajaraman, D. Whiteson, and F. Yu, Collider, Signals of maximal flavor violation: Same-sign leptons from same-sign tops at the tevatron, Phys. Rev. D 78, 033003 (2008).

[63] R. S. Chivukula, E. H. Simmons, and N. Vignaroli, Samesign dileptons from colored scalars in the flavorful topcoloron model, Phys. Rev. D 88, 034006 (2013).

[64] H. Zhang, E. L. Berger, Q. H. Cao, C. R. Chen, and G. Shaughnessy, Color sextet vector bosons and samesign top quark pairs at the LHC, Phys. Lett. B 696, 68 (2011).

[65] D. Atwood, S. K. Gupta, and A. Soni, Same-sign tops: A powerful diagnostic test for models of new physics, J. High Energy Phys. 04 (2013) 035.

[66] M. Aaboud et al. (ATLAS Collaboration), Measurements of gluon-gluon fusion and vector-boson fusion Higgs boson production cross-sections in the $H \rightarrow W W^{*} \rightarrow e \nu \mu \nu$ decay channel in $p p$ collisions at $\sqrt{s}=13 \mathrm{TeV}$ with the ATLAS detector, Phys. Lett. B 789, 508 (2019).

[67] G. Aad et al. (ATLAS Collaboration), Measurement of the production cross section for a Higgs boson in association with a vector boson in the $H \rightarrow W W^{*} \rightarrow \ell \nu \ell \nu$ channel in $p p$ collisions at $\sqrt{s}=13 \mathrm{TeV}$ with the ATLAS detector, arXiv:1903.10052. 
[68] A. M. Sirunyan et al. (CMS Collaboration), Measurements of properties of the Higgs boson decaying to a $W$ boson pair in pp collisions at $\sqrt{s}=13 \mathrm{TeV}$, Phys. Lett. B 791, 96 (2019).
[69] A. M. Sirunyan et al. (CMS Collaboration), Search for the associated production of the Higgs boson and a vector boson in proton-proton collisions at $\sqrt{s}=13 \mathrm{TeV}$ via Higgs boson decays to $\tau$ leptons, arXiv:1809.03590. 